\title{
$-$
}

\section{Nodal Solutions for Supercritical Laplace Equations}

\author{
Francesca Dalbono $^{1}$, Matteo Franca ${ }^{2}$ \\ 1 Dipartimento di Matematica e Informatica, Università degli Studi di Palermo, Via Archirafi 34, 90123 \\ Palermo, Italy. \\ 2 Dipartimento di Scienze Matematiche, Università Politecnica delle Marche, Via Brecce Bianche 1, 60131 \\ Ancona, Italy. E-mail: franca@ dipmat.univpm.it
}

Received: 8 September 2015 / Accepted: 17 September 2015

(C) Springer-Verlag Berlin Heidelberg 2015

Abstract: In this paper we study radial solutions for the following equation

$$
\Delta u(x)+f(u(x),|x|)=0,
$$

where $x \in \mathbb{R}^{n}, n>2, f$ is subcritical for $r$ small and $u$ large and supercritical for $r$ large and $u$ small, with respect to the Sobolev critical exponent $2^{*}=\frac{2 n}{n-2}$. The solutions are classified and characterized by their asymptotic behaviour and nodal properties. In an appropriate super-linear setting, we give an asymptotic condition sufficient to guarantee the existence of at least one ground state with fast decay with exactly $j$ zeroes for any $j \geq 0$. Under the same assumptions, we also find uncountably many ground states with slow decay, singular ground states with fast decay and singular ground states with slow decay, all of them with exactly $j$ zeroes. Our approach, based on Fowler transformation and invariant manifold theory, enables us to deal with a wide family of potentials allowing spatial inhomogeneity and a quite general dependence on $u$. In particular, for the Matukuma-type potential, we show a kind of structural stability.

\section{Introduction}

In this paper we focus on radial solutions for Laplacian equations of the form

$$
\Delta u(x)+f(u(x),|x|)=0,
$$

where $x \in \mathbb{R}^{n}, n>2, f$ is a suitable locally Lipschitz continuous function, satisfying $f(0, r)=0$, super-linear in $u$. Since we just deal with radial solutions, we set $r=|x|$ and we consider the equivalent singular O.D.E.

$$
\left(u^{\prime} r^{n-1}\right)^{\prime}+f(u, r) r^{n-1}=0, \quad r \in(0, \infty),
$$

F. Dalbono supported by G.N.A.M.P.A.

M. Franca supported by G.N.A.M.P.A.-INdAM (Italy) and MURST (Italy).

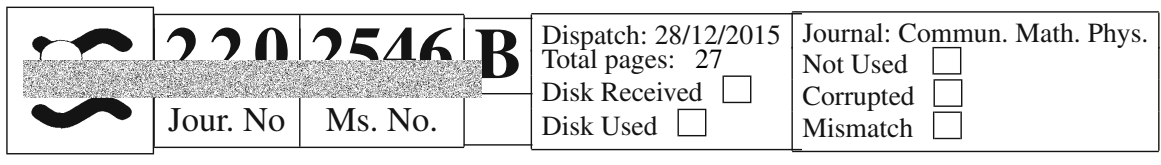


where, abusing the notation, we have set $u(r)=u(x)$ for $|x|=r$, and where " " " denotes the differentiation with respect to $r$. We are concerned with the study of asymptotic behaviour and nodal properties of the solutions to equation (1.2). The interest in equations of the family (1.2) started long ago from nonlinearities $f$ of the form

$$
f(u, r)=k(r) u|u|^{q-2}, \quad q>2,
$$

where $k$ is a differentiable positive function. The structure of solutions to this class of equations has been intensively studied in the literature, see e.g. $[1,5,6,13,16,20,26,28$, 30,32,39-42] and references therein.

It has been shown that, under very weak assumptions, solutions of (1.2) exhibit two behaviors as $r \rightarrow 0$ and as $r \rightarrow \infty$. Namely, $u(r)$ may be a regular solution, i.e., $u(0)=d \neq 0$ and $u^{\prime}(0)=0$, or a singular solution, i.e., $\lim _{r \rightarrow 0} u(r)= \pm \infty$; similarly, $u(r)$ may be a fast decay solution, i.e., $\lim _{r \rightarrow \infty} u(r) r^{n-2}=L \neq 0$, or a slow decay solution, i.e., $\lim _{r \rightarrow \infty} u(r) r^{n-2}= \pm \infty$. We remark that, in many situations, it is possible to specify in more detail the behavior of singular and slow decay solutions: e.g., if $k(r)=c r^{\delta}, \delta>-2, c>0$, then $u(r) r^{\frac{2+\delta}{q-2}} \rightarrow C$ as $r \rightarrow 0$ or as $r \rightarrow+\infty$ respectively, where $C$ is a computable constant (for more details, see Sect. 2, and [1, 13,16,17], among others).

Solutions of (1.2) are classified as ground states (G.S.) and singular ground states (S.G.S.). By G.S. we mean a regular solution $u(r)$ defined for any $r \geq 0$ such that $\lim _{r \rightarrow \infty} u(r)=0$, while a S.G.S is a singular solution $u(r)$ which is defined for any $r>0$ and goes to 0 as $r \rightarrow+\infty$.

It is well known that the structure of positive solutions of (1.2) changes drastically when the exponent $q$ in (1.3) passes through some critical values related to the behaviour of the function $k$, due to the interaction between the exponent and the asymptotic behavior of $k$. In particular, when $k$ is a constant, the critical value is given by the Sobolev critical exponent $2^{*}:=\frac{2 n}{n-2}$, while if $k(r)=r^{\delta}$, it becomes $2_{\delta}^{*}=2 \frac{\delta+n}{n-2}=\frac{2 \delta}{n-2}+2^{*}$. Such a phenomenon is better explained and incorporated in a more general framework by the introduction of the concept of natural dimension, see e.g. [37]. A further critical value which is relevant for the asymptotic behaviour of singular solutions is $2_{*}:=\frac{2(n-1)}{n-2}$. In this paper we are interested in nonlinearities $f$ which are subcritical for $u$ large and $r$ small, and supercritical for $u$ small and $r$ large.

The prototypical nonlinearity we are interested in is $(1.3)$, where $k(r)>0, k(r)$ differentiable for $r>0$ and such that

$$
k(r)=A r^{s}+o\left(r^{s}\right) \text { at } r=0 \text { and } k(r)=B r^{l}+o\left(r^{l}\right) \text { at } r=\infty,
$$

for suitable values of the powers $l, s$. We also devote our attention to the study of the following classes of nonlinearities:

$$
f(u, r)=k(r) \times \begin{cases}u|u|^{q_{1}-2}, & \text { if }|u| \geq 1 \\ u|u|^{q_{2}-2}, & \text { if }|u| \leq 1\end{cases}
$$

with $q_{1}, q_{2}>2$,

$$
f(u, r)=k_{2}(r) \frac{u|u|^{q_{2}-2}}{1+k_{1}(r)|u|^{q_{1}}},
$$

with $q_{1}>1, q_{2}-q_{1}>2$, and

$$
f(u, r)=k_{1}(r) u|u|^{q_{1}-2}+k_{2}(r) u|u|^{q_{2}-2},
$$

\begin{tabular}{|l|l|l|l|l|l|}
\hline 220 & 254.6 & $\mathbf{B}$ & $\begin{array}{l}\text { Dispatch: 28/12/2015 } \\
\text { Total pages: 27 } \\
\text { Disk Received } \square \\
\text { Disk Used } \square\end{array}$ & $\begin{array}{l}\text { Journal: Commun. Math. Phys. } \\
\text { Not Used } \square \\
\text { Corrupted } \square \\
\text { Mismatch } \square\end{array}$ \\
\hline
\end{tabular}


with $q_{1}, q_{2}>2$. In all the cases (1.5), (1.6) and (1.7), we assume that the functions $k, k_{i}$ satisfy (1.4), and some further conditions.

The aim of this paper consists in completing the analysis performed in [16] (see also $[5,7,20,42]$ ) with information concerning the nodal properties of solutions. Our main result, Theorem 2.4, gives sufficient conditions to have the following structure for positive and nodal solutions.

Mix Let $u(r, d)$ be the regular solution of (1.2) satisfying the initial condition

$$
u(0)=d>0, \quad u^{\prime}(0)=0 .
$$

Then, there is a sequence $0=d_{0}<d_{0}^{*} \leq d_{1}<d_{1}^{*} \leq d_{2}<d_{2}^{*} \leq \cdots \leq d_{j}<$ $d_{j}^{*} \rightarrow+\infty$ as $j \rightarrow+\infty$, such that $u\left(r, d_{j}^{*}\right)$ are G.S. with fast decay with exactly $j$ non-degenerate zeroes. In particular, $u\left(r, d_{0}^{*}\right)$ is a positive G.S. with fast decay. Moreover, $u(r, d)$ is a positive G.S. with s.d. for any $d \in\left(0, d_{0}^{*}\right)$, while $u(r, d)$ is a G.S. with s.d. with exactly $j$ non-degenerate zeroes whenever $d \in\left(d_{j}, d_{j}^{*}\right)$, for any $j \geq 1$.

Let $v(r, L)$ be the fast decay solution of (1.2) such that

$$
\lim _{r \rightarrow \infty} v(r, L) r^{n-2}=L
$$

Then, there is an increasing sequence $0=L_{0}<L_{0}^{*} \leq L_{1}<L_{1}^{*} \leq L_{2}<L_{2}^{*} \leq$ $\cdots \leq L_{j}<L_{j}^{*} \rightarrow+\infty$ as $j \rightarrow+\infty$, such that $v\left(r, L_{j}^{*}\right)$ are G.S. with fast decay with exactly $j$ non-degenerate zeroes. Moreover, $v(r, L)$ is a positive S.G.S. with f.d. for any $L \in\left(0, L_{0}^{*}\right)$, while $v(r, L)$ is a S.G.S. with f.d. with exactly $j$ non-degenerate zeroes whenever $L \in\left(L_{j}, L_{j}^{*}\right)$, for any $j \geq 1$.

For any $k \geq 0$ there are uncountably many singular solutions $u_{k}(r)$ of (1.2) which have slow decay and have exactly $k$ non-degenerate zeroes. In particular, there are uncountably many positive S.G.S. with slow decay $u_{0}(r)$.

We emphasize that with the same argument we can obviously obtain the symmetric case, i.e. regular nodal solutions $u$ with negative initial data, and fast decay nodal solutions $v$ which are negative for $r$ large.

In the case of potentials of the form (1.3), we choose the powers in order to handle nonlinearities which are supercritical for $r$ large and subcritical for $r$ small. A particularly relevant example is given by the so called Matukuma equation (cf., among others [34, $35]$ ), which finds application in astrophysics ( $u$ represents the gravitational potential in a globular cluster), i.e.,

$$
k(r)=\frac{1}{r^{a}+r^{b}}, \quad \text { where }-2<a<\frac{n-2}{2}\left(q-2^{*}\right)<b<(n-2)(q-2 *) .
$$

Potentials of type (1.3) are the most studied in the literature: in [42], the authors proved the structure result, but just for positive and regular solutions; this result was extended to the $p$-Laplace case in [20], and then completed by the analysis of positive singular solutions in [16].

It is worth noticing that Yanagida in [39], using the monotonicity properties of the first zero $R(d)$ of the solution $u(r, d)$, proved the following theorem (we became aware of this paper just after this article was completed).

\begin{tabular}{|l|l|l|l|l|l|}
\hline 220 & 2546 & $\mathbf{B}$ & $\begin{array}{l}\text { Dispatch: 28/12/2015 } \\
\text { Total pages: 27 } \\
\text { Disk Received } \\
\text { Disk Used } \square\end{array}$ & $\begin{array}{l}\text { Journal: Commun. Math. Phys. } \\
\text { Not Used } \square \\
\text { Corrupted } \square \\
\text { Mismatch } \square\end{array}$ \\
\hline
\end{tabular}


Theorem A. [39] Consider (1.2) with $f$ satisfying (1.3), (1.4) and $l<\frac{n-2}{2}\left(q-2^{*}\right)<s$. Assume that $\frac{r k^{\prime}(r)}{k(r)}$ is decreasing, but not identically constant. Then, all the regular or fast decay solutions of (1.2) have a structure of type Mix, $d_{j}^{*}=d_{j+1}$ and $L_{j}^{*}=L_{j+1}$, for any $j \geq 0$.

Note that Theorem A applies e.g. to the case (1.8). Observe, moreover, that in [39] singular solutions are not considered; their analysis has been recently improved in [6, Theorem 1.2], proving the existence of singular-fast decay solutions and of singularslow decay solutions, which are positive or have one zero, but with the restriction $s \leq$ $q(n-2)-n$.

Here, we extend the result to singular-slow decay solutions with any number of zeroes. We further restrict the range of $s$ by imposing $s<(n-2)(q-2 *)$; such a requirement allows us to improve the estimates on the asymptotic behaviour of singular solutions. A further relevant contribution we provide in this paper consists in proving the nodal result without any monotonicity condition on $\frac{r k^{\prime}(r)}{k(r)}$, although we get $d_{j}^{*} \leq d_{j+1}$ and $L_{j}^{*} \leq L_{j+1}$

Since we just assume the asymptotic conditions (1.4), we can interpret our contribution as the following structural stability result:

Consider $f$ satisfying (1.3) with $k(r)=k_{1}(r)+k_{2}(r)$, where $k_{1}(r)$ is as in Theorem A, and $k_{2}(r)$ is a nonnegative function such that $k_{2}(r) \equiv 0$ for any $r \in([0,1 / M] \cup[M,+\infty))$, for a certain $M>0$. Then, all the solutions of (1.2) have a structure of type Mix.

So, roughly speaking, perturbations do not affect the existence result of Theorem A for positive and nodal solutions, but they may affect the "uniqueness" of these nodal solutions.

We wish to remark that also in the papers [28,31,41] no monotonicity condition is required to get nodal solutions to (1.2) under potentials of the form (1.3). More precisely, under an asymptotic condition of type (1.4), the authors of these papers obtain regularfast decay solutions to (1.2), but no information concerning slow decay or singular solutions is furnished.

Following [6], we denote by $\mathcal{T}(u):=\int_{\mathbb{R}^{n}} f(u(x),|x|) d x$ the so called total curvature associated with $u$, which is relevant for associated problems in differential geometry. According to [11, Remark 1.4], it is worth stressing that, in the range of parameters considered, $\mathcal{T}(u)$ is finite whenever $u$ has fast decay, independently of the behaviour of $u$ (either regular or singular) at $r=0$. Thus, singular solutions are "physical". However, $\mathcal{T}(u)$ is infinite if $u$ has slow decay. An analogous phenomenon occurs in the Matukuma equation: in this context $\mathcal{T}(u)$ represents the total mass (cf., among others [34,35]).

In case of potentials of the form (1.5) and (1.6), we choose the powers in order to deal with nonlinearities, which are supercritical for $u$ small and subcritical for $u$ large, with respect to the Sobolev critical exponent $2^{*}$. In this setting, we quote [7] and [10], dealing with the autonomous case, where the part of Mix concerning positive solutions is proved. Our work completes this analysis by studying the nodal properties and allowing spatial dependence.

This paper has been inspired by [16], which introduces a unifying approach able to handle simultaneously nonlinearities of the form (1.3), (1.5), (1.6) and (1.7). In fact, in [16] structure Mix is obtained, but just for positive solutions, in the more general $p$ Laplace context. Here, we extend the analysis to nodal solutions, maintaining the main assumptions on the potentials, but we restrict to the classical Laplace case to clarify the argument and to avoid some major technical difficulties (arising especially in the $p>2$ case). 
We now state the following consequence of our main Theorem (2.4).

Corollary 1.2. Let us define $\lambda(q):=\frac{n-2}{2}\left(q-2^{*}\right)$ and $\eta(q):=(n-2)(q-2 *)$. Assume either that

1. $f$ is of type (1.3), $q>2$, $k$ satisfies (1.4) where $A, B>0,-2<l<\lambda(q)<s<$ $\eta(q)$.

2. $f$ is of type (1.5), $q_{1}, q_{2}>2, k$ satisfies (1.4), where $A, B>0, s, l>-2, \lambda\left(q_{1}\right)<$ $s<\eta\left(q_{1}\right)$, and $l<\lambda\left(q_{2}\right)$.

3. $f$ is of type (1.6), $q_{1}>1, q_{2}-q_{1}>2, k_{i}$ satisfy

$$
k_{i}(r)=A_{i} r^{s_{i}}+o\left(r^{s_{i}}\right) \text { at } r=0 \text { and } k_{i}(r)=B_{i} r^{l_{i}}+o\left(r^{l_{i}}\right) \text { at } r=\infty \text {, }
$$

where $A_{i}, B_{i}>0$ for every $i \in\{1,2\}, l_{2}>-2, s_{2}-s_{1}>-2$,

$$
\begin{gathered}
s_{2}+2>\frac{q_{2}-2}{q_{1}} s_{1}, \quad l_{1}<\frac{\left(2+l_{2}\right) q_{1}}{q_{2}-2}, \\
\lambda\left(q_{2}-q_{1}\right)<s_{2}-s_{1}<\eta\left(q_{2}-q_{1}\right), \quad l_{2}<\lambda\left(q_{2}\right) .
\end{gathered}
$$

4. $f$ is of type (1.7), $q_{1}>2, q_{2}>2, k_{i}$ satisfies (1.9), where $A_{i}, B_{i}>0, s_{i}, l_{i}>-2$ for every $i \in\{1,2\}$ and

$$
\begin{gathered}
\max \left\{\lambda\left(q_{1}\right)-s_{1} ; \lambda\left(q_{2}\right)-s_{2}\right\}<0<\min \left\{\lambda\left(q_{1}\right)-l_{1} ; \lambda\left(q_{2}\right)-l_{2}\right\}, \\
\max \left\{\eta\left(q_{1}\right)-s_{1} ; \eta\left(q_{2}\right)-s_{2}\right\}>0 .
\end{gathered}
$$

Assume further that all the functions $k, k_{i}$ defined above are positive and Lipschitz for $r>0$, then all the solutions of (1.2) have a structure of type Mix.

The meaning of the restrictions on the parameters $l, l_{i}, s, s_{i}, q, q_{i}$ will be shortly clarified at Remark 4.1.

Summing up, we propose a unified approach which allows us to deal with the case where $f$ is subcritical for $u$ large and $r$ small, and supercritical for $u$ small and $r$ large, so that the change on the criticality of the potential may be due either to the dependence on $u$ or to the dependence on $|x|$, or to a mixture of both. In this way, we complete the literature regarding nonlinearities $f$ of the form (1.3) with a discussion of nodal singular solutions, and we improve the literature regarding nonlinearities $f$ of the form (1.5), (1.6) and (1.7) with the entire study of nodal solutions (compare, in particular, with $[6,7,16,39])$, and by weakening the assumptions on $f$.

Concerning the methods, in this paper we use Fowler transformation to convert (1.2) to a non-autonomous two-dimensional and to an autonomous three-dimensional dynamical system (cf. (2.2) and (2.6)-(2.7) below, respectively), which can be treated by means of invariant manifold theory. Multiplicity results arise by combining these techniques with the notion of rotation or winding number (cf. (3.5) below). We observe that similar approaches have been followed, among others, in [27] and [2], where multiplicity of solutions have been achieved for suitable autonomous problems of the form (1.1).

We complete the paper with a brief analysis of the critical case

$$
f(u, r)=\sum_{i=1}^{j} c_{i} r^{\delta_{i}} u|u|^{q_{i}-2}, \quad c_{i} \geq 0, \quad \delta_{i}=\frac{n-2}{2}\left(q_{i}-2^{*}\right) .
$$

The idea to include this case originated from [6], devoted to the study of (1.2)-(1.3), involving critical nonlinearities as well as nonlinearities that are supercritical for $r$ large and subcritical for $r$ small. We extend the comparison with [6] by treating also the critical

\begin{tabular}{|l|l|l|l|l|l|}
\hline 220 & 2546 & $\mathbf{B}$ & $\begin{array}{l}\text { Dispatch: 28/12/2015 } \\
\text { Total pages: 27 } \\
\text { Disk Received } \\
\text { Disk Used } \square\end{array}$ & $\begin{array}{l}\text { Journal: Commun. Math. Phys. } \\
\text { Not Used } \square \\
\text { Corrupted } \square \\
\text { Mismatch } \square\end{array}$ \\
\hline
\end{tabular}


case. Even in the general setting (1.14), we can draw all the trajectories and establish a correspondence between initial values and associated finite total curvature, extending Theorem 1.1 in [6]. In particular, by Fowler transformation we easily get the following result:

Remark 1.3. Assume $f$ as in (1.14), then all the regular solutions are positive, and the total curvature $\mathcal{T}(d):=\mathcal{T}(u(r, d))$ satisfies

$$
\mathcal{T}(d):=\int_{\mathbb{R}^{n}} f(u(x, d),|x|) d x=d^{-1} \mathcal{T}(1) .
$$

In particular, for any $T>0$ there is a unique $d=\frac{\mathcal{T}(1)}{T}$ such that $\mathcal{T}(d)=T$.

Moreover, if $d \neq d_{0}$ there is a unique intersection $R(d)$ between $u(r, d)$ and $u\left(r, d_{0}\right)$, and $\lim _{d \rightarrow 0} R(d)=+\infty, \lim _{d \rightarrow+\infty} R(d)=0, R(d)$ is monotone decreasing.

Restricting to the critical situation considered in [6] with nonlinearities of the form $f(u, r)=c_{1} r^{\delta_{1}} u|u|^{q_{1}-2}$, we notice that the solutions of (1.2) are explicitly known (even in the $p$-Laplace context), see e.g. [15] for the case $\delta_{1}=0$. Concerning the case $\delta_{1} \neq 0$, it can be reduced to the $\delta_{1}=0$ case, by applying the natural dimension change of variable, see [37].

Throughout the paper, we assume that $0 \in \mathbb{N}$.

The paper is organized as follows: in Sect. 2 we introduce Fowler transformation to convert Eq. (1.2) into a system, we review some basic facts concerning the new formulation of our problem and we state the general result Theorem 2.4; in Sect. 3 we prove Theorem 2.4; in Sect. 4 we deduce Corollary 1.2 from Theorem 2.4 and we prove Remark 1.3.

\section{Basic Results on Fowler Transformation}

We devote the first part of this Section to introduce a change of variables known as Fowler transformation, see [12], which allows to pass from (1.2) to a two-dimensional dynamical system. Let us define

$$
\begin{aligned}
\alpha_{l} & =\frac{2}{l-2}, \quad \gamma_{l}=\alpha_{l}-(n-2), \quad l>2 \\
x_{l} & =u(r) r^{\alpha_{l}} \quad y_{l}=u^{\prime}(r) r^{\alpha_{l}+1} \quad r=e^{t} .
\end{aligned}
$$

The new variables $x_{l}, y_{l}$ differ from the given ones $u, u^{\prime}$ in the presence of weight terms, which will help us to determine the asymptotic behaviors. Applying (2.1), we can rewrite (1.2) as the following two-dimensional system

$$
\left(\begin{array}{c}
\dot{x}_{l} \\
\dot{y}_{l}
\end{array}\right)=\left(\begin{array}{cc}
\alpha_{l} & 1 \\
0 & \gamma_{l}
\end{array}\right)\left(\begin{array}{l}
x_{l} \\
y_{l}
\end{array}\right)+\left(\begin{array}{c}
0 \\
-g_{l}\left(x_{l}, t\right)
\end{array}\right)
$$

which is as smooth as $g_{l}$. Here and later "." stands for $\frac{d}{d t}$, and

$$
g_{l}(x, t):=f\left(x \exp \left(-\alpha_{l} t\right), \exp (t)\right) e^{\left(\alpha_{l}+2\right) t} .
$$

We begin our discussion reviewing some well known facts concerning the $t$-independent case $g_{l}(x, t) \equiv g_{l}(x)$. In particular, we consider $f(u, r)=r^{\delta} u|u|^{q-2}$, with $q>2$ and $\delta>-2$ : in this case,

$$
l=2 \frac{q+\delta}{2+\delta} \quad \Longrightarrow \quad g_{l}(x, t)=x|x|^{q-2},
$$

\begin{tabular}{|l|l|l|l|l|l|}
\hline 220 & 254.6 & $\mathbf{B}$ & $\begin{array}{l}\text { Dispatch: 28/12/2015 } \\
\text { Total pages: 27 } \\
\text { Disk Received } \square \\
\text { Disk Used } \square\end{array}$ & $\begin{array}{l}\text { Journal: Commun. Math. Phys. } \\
\text { Not Used } \square \\
\text { Corrupted } \square \\
\text { Mismatch } \square\end{array}$ \\
\hline
\end{tabular}


so (2.2) is autonomous, and we have removed the singularity in $r$ from (1.2). Note that if $\delta=0$, then $l=q$.

Using invariant manifold theory $[13,14,17]$, we see that if $l>2_{*}$, the origin of (2.2) admits an unstable manifold $M^{u}$ and a stable manifold $M^{s}$.

Remark 2.1. In the origin the unstable manifold $M^{u}$ is tangent to the $x$-axis, while the stable manifold $M^{s}$ is tangent to the line $y=-(n-2) x$.

The manifold $M^{u}$ (and $M^{s}$ ) is split by the origin in two connected components: one which leaves the origin and enters $x>0$, say $M^{u,+}$ (respectively $M^{s,+}$ ), and the other that enters $x<0$, say $M^{u,-}$ (respectively $M^{s,-}$ ).

Furthermore, there are a unique critical point $\boldsymbol{P}^{+}=\left(P_{x}^{+}, P_{y}^{+}\right)$in the $x>0$ semiplane, and a unique one in the $x<0$ semiplane, say $\boldsymbol{P}^{-}=\left(P_{x}^{-}, P_{y}^{-}\right)$; they are both stable if $l>2^{*}$, unstable if $2_{*}<l<2^{*}$ and centers if $l=2^{*}$.

Remark 2.2. Assume that $g_{l}(x, t)=x|x|^{q-2}$. Denote by $\boldsymbol{X}_{l}(t ; \tau, \boldsymbol{Q}):=\left(x_{l}(t ; \tau, \boldsymbol{Q})\right.$, $\left.y_{l}(t ; \tau, \boldsymbol{Q})\right)$ the trajectory of (2.2) satisfying the initial condition $\boldsymbol{X}_{l}(\tau)=\boldsymbol{Q} \in \mathbb{R}^{2}$. Let $u(r)$ be the corresponding solution of (1.2), then

$$
\begin{aligned}
& u(r) \text { is a regular solution } \Longleftrightarrow Q \in M^{u}, \\
& u(r) \text { is a fast decay solution } \Longleftrightarrow Q \in M^{s} .
\end{aligned}
$$

Moreover, if $\boldsymbol{Q} \in M^{u,+}$, then $u(0)=d>0$, while if $\boldsymbol{Q} \in M^{u,-}$, then $d<0$; similarly, if $\boldsymbol{Q} \in M^{s,+}$, then $\lim _{r \rightarrow \infty} u(r) r^{n-2}=L>0$, while if $\boldsymbol{Q} \in M^{s,-}$, then $L<0$.

Using the Pohozaev identity, see e.g. [13,14], it can be shown that the phase portrait is as in Fig. 1 when $g_{l}(x, t)=x|x|^{q-2}$. From the picture, we can classify completely positive and nodal solutions. As observed in [14], stable and unstable manifolds exhibit the same features sketched in Fig. 1, whenever $g_{l}(x, t)$ is $t$-independent, i.e. $g_{l}(x, t) \equiv g_{l}(x)$, and satisfies the following super-linear condition:

$\boldsymbol{G}_{\mathbf{0}} g_{l}(x)$ is a locally Lipschitz function such that $x g_{l}(x)>0$ for $x \neq 0, \mathfrak{G}_{l}(x)=$ $g_{l}(x) / x$ is decreasing for $x<0$ and increasing for $x>0$, and satisfies $\mathfrak{G}_{l}(0)=0$, $\lim _{|x| \rightarrow \infty} \mathfrak{G}_{l}(x)=\infty$.

Remark 2.3. We observe that in $[13,14]$ the whole analysis is developed just for $M^{u,+}$ and $M^{s,+}$. However, if $g_{l}(x)$ is odd as in Remark 2.2 (i.e. $f(u, r)$ is odd in $u$ ), then $M^{u}$ and $M^{s}$ are symmetric with respect to the origin, e.g. if $\boldsymbol{Q} \in M^{u,+}$, then $-\boldsymbol{Q} \in M^{u,-}$, and analogously for $M^{s}$. If $g_{l}$ is not odd but satisfies $\boldsymbol{G}_{\mathbf{0}}$, it is trivial to check that $M^{u,-}$ is a slight deformation of $\bar{M}^{u,-}=\left\{-\boldsymbol{Q} \mid \boldsymbol{Q} \in M^{u,+}\right\}$, and similarly for $M^{s,-}$.

We are now interested in describing the structure of the set of solutions of the general non-autonomous Eq. (2.2). We emphasize that our approach is based on the fact that (2.2) is locally Lipschitz continuous, and, in this setting, invariant manifold theory tools can be used. However, we wish to remark that, in absence of Lipschitz continuity assumptions, the results concerning positive solutions can still be proved using a more technical dynamical approach relying on Wazewski's principle, see [16], or using a completely different approach, as the one adopted in [20]. However, in [20] the nonlinearities considered are just of type (1.3) and there is no discussion concerning singular solutions.

In order to extend the concept of stable and unstable manifolds and to present our main result, we introduce further assumptions which establish an asymptotic relation between the given non-autonomous problem and suitable autonomous ones (cf. $[14,16,18]$ for similar assumptions).

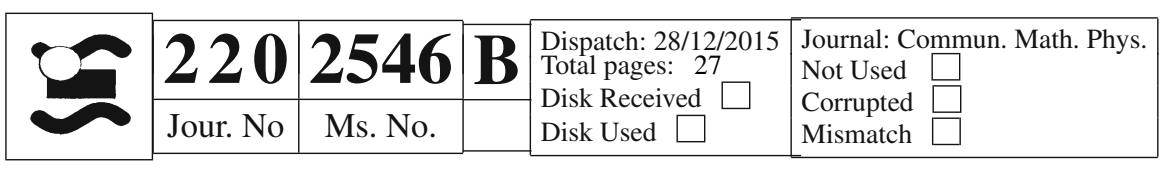



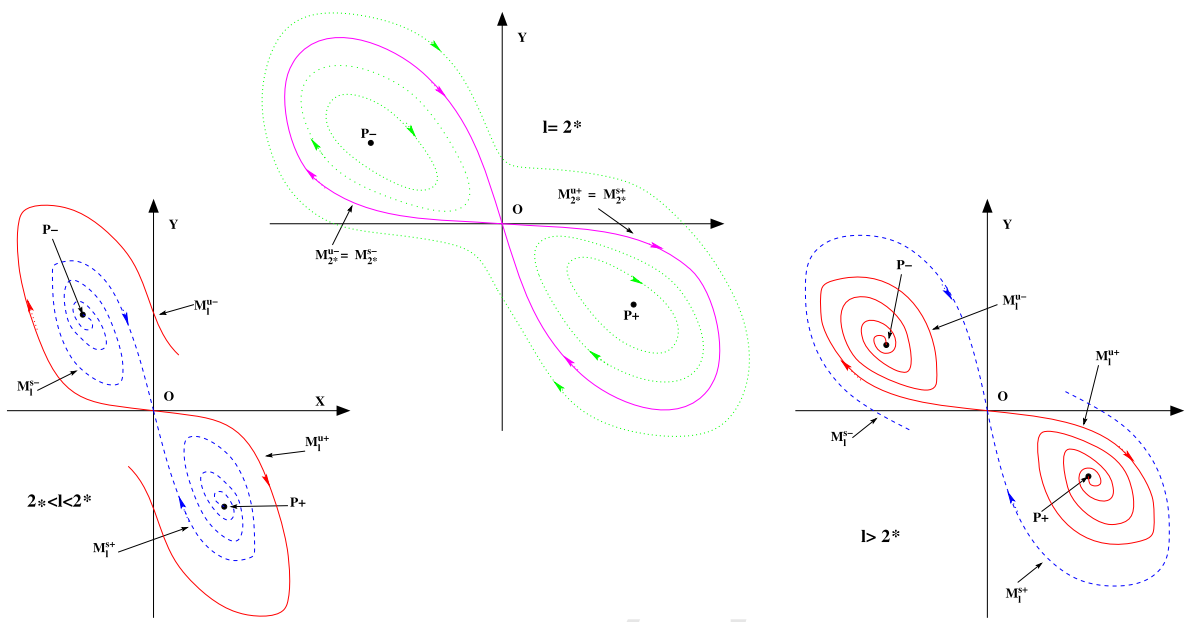

Fig. 1. Sketch of the phase portrait of (2.2), when $g_{l}(x, t)$ is $t$-independent and satisfies $\boldsymbol{G}_{\mathbf{0}}$. The unstable manifolds $M^{u}$ are the red solid lines, the stable manifolds $M^{s}$ are the blue dashed lines, apart from the critical case where they coincide and they are represented by a solid magenta line. In the critical case we have also represented some further dashed and green trajectories corresponding to S.G.S. with slow decay (levels of negative $H$ ) and to sign changing solutions (levels of positive $H$ ) (colour figure online)

$\boldsymbol{G}_{\mathbf{1}}$ There is $l>2$ such that $g_{l}(x, t)$ satisfies $\boldsymbol{G}_{\mathbf{0}}$ for any $t \in \mathbb{R}$.

$\boldsymbol{G}_{\boldsymbol{u}}$ There is $l_{u}>2 *$ such that for any $x>0$ the function $g_{l_{u}}(x, t)$ converges to a $t$-independent function $g_{l_{u}}^{-\infty}(x) \not \equiv 0$ as $t \rightarrow-\infty$, uniformly on compact intervals. The function $g_{l_{u}}^{-\infty}(x)$ satisfies $\boldsymbol{G}_{\mathbf{0}}$.

Moreover, $g_{l_{u}}(x, t)$ is differentiable in $t$ in a neighbourhood of $t=-\infty$, for any $x$, and there is $\varpi>0$ such that $\lim _{t \rightarrow-\infty} e^{-\varpi t} \frac{\partial}{\partial t} g_{l_{u}}(x, t)=0$.

$\boldsymbol{G}_{\boldsymbol{s}}$ There is $l_{s}>2 *$ such that for any $x>0$ the function $g_{l_{s}}(x, t)$ converges to a $t$-independent function $g_{l_{s}}^{+\infty}(x) \not \equiv 0$ as $t \rightarrow+\infty$, uniformly on compact intervals. The function $g_{l_{s}}^{+\infty}(x)$ satisfies $\boldsymbol{G}_{\mathbf{0}}$.

Moreover, $g_{l_{s}}(x, t)$ is differentiable in $t$ in a neighbourhood of $t=+\infty$, for any $x$, and there is $\varpi>0$ such that $\lim _{t \rightarrow \infty} e^{\varpi t} \frac{\partial}{\partial t} g_{l_{s}}(x, t)=0$.

We emphasize that if $\boldsymbol{G}_{\mathbf{1}}$ holds for a certain $l>2$, then it holds for any $L>2$ (see [14]). Now we are ready to state the main result of the paper.

Theorem 2.4. Assume that $u f(u, r)>0$ for $u \neq 0$ and $f(0, r)=0$, with $f(u, r)$ locally Lipschitz in $u \in \mathbb{R}$ and differentiable in $r \in(0,+\infty)$. Suppose that there exists a continuous function $h:[0,+\infty) \mapsto[0,+\infty)$ such that

$$
\int_{0}^{x} \frac{\partial}{\partial r} f(u, r) d u \leq h(r) \int_{0}^{x} f(u, r) d u \quad \forall(x, r) \in \mathbb{R} \times(0,+\infty) .
$$

Moreover, assume that $\boldsymbol{G}_{\mathbf{1}}, \boldsymbol{G}_{\boldsymbol{u}}$ and $\boldsymbol{G}_{\boldsymbol{s}}$ hold with

$$
2_{*}<l_{u}<2^{*}<l_{s} .
$$

Then, all the solutions of (1.2) have a structure of type Mix.

\begin{tabular}{|l|l|l|l|l|l|}
\hline 220 & $\mathbf{2 5 4 6}$ & $\mathbf{B}$ & $\begin{array}{l}\text { Dispatch: 28/12/2015 } \\
\text { Total pages: 27 } \\
\text { Disk Received } \\
\text { Disk Used } \square\end{array}$ & $\begin{array}{l}\text { Journal: Commun. Math. Phys. } \\
\text { Not Used } \square \\
\text { Corrupted } \square \\
\text { Mismatch } \square\end{array}$ \\
\hline
\end{tabular}


In particular, our system has a subcritical autonomous behaviour as $t$ tends to $-\infty$ and a supercritical autonomous behaviour as $t$ tends to $+\infty$. We are able to draw the picture of the phase portraits in the asymptotic autonomous cases. The key idea to prove the result is to overlap and intersect in a suitable way stable and unstable manifolds.

Remark 2.5. Assumption (2.4) is a well-known condition ensuring the continuability of the solutions of any Cauchy problem associated with (1.2) in $r>0$. The proof of the global continuability result is based on an appropriate energy estimate combined with the Gronwall's Lemma (cf., among others [4]).

According to [8] and [36, Sect. 2.1], we point out that both the differentiability condition in the variable $r$ and assumption (2.4) can be omitted in case of nonlinearities of the form $f(u, r):=k(r) G^{\prime}(u)$, where $k$ is a positive and Lipschitz function in $[0,+\infty)$, and $G \in C^{1}(\mathbb{R})$ with $\inf _{\mathbb{R}} G>-\infty$. An analogous remark holds true for nonlinearities of the form (1.6), and it can be deduced by the approximation procedure developed in [36, Sect. 2.1]. This justifies the absence of assumption (2.4) in Corollary 1.2.

Assume the validity of condition $\boldsymbol{G}_{\mathbf{1}}$ in the rest of the paper.

We now focus on the study of the properties of the two-dimensional system (2.2). Inspired by $[2,18,27]$, we rewrite $(2.2)$ as an equivalent three-dimensional autonomous system, adding the variable $z=e^{\varpi t}$ :

$$
\left(\begin{array}{c}
\dot{x}_{l_{u}} \\
\dot{y}_{l_{u}} \\
\dot{z}
\end{array}\right)=\left(\begin{array}{ccc}
\alpha_{l_{u}} & 1 & 0 \\
0 & \gamma_{l_{u}} & 0 \\
0 & 0 & \varpi
\end{array}\right)\left(\begin{array}{c}
x_{l_{u}} \\
y_{l_{u}} \\
z
\end{array}\right)+-g_{l_{u}}\left(x_{l_{u}}, \frac{\ln (z)}{\omega}\right) .
$$

Observe that all the trajectories converge to the $z=0$ plane as $t \rightarrow-\infty$, so (2.6) is useful to investigate the asymptotic behavior in the past. If we assume $\boldsymbol{G}_{\boldsymbol{u}}$, the origin admits a two-dimensional unstable manifold denoted by $\boldsymbol{W}^{\boldsymbol{u}}$. From standard argument of dynamical system theory, we see that the set $\tilde{W}_{l_{u}}^{u}(\tau)=\boldsymbol{W}^{\boldsymbol{u}} \cap\left\{z=e^{\varpi \tau}\right\}$ is a one-dimensional manifold, for any $\tau \in \mathbb{R}$. Note that $\tilde{W}_{l_{u}}^{u}(-\infty):=\boldsymbol{W}^{\boldsymbol{u}} \cap\{z=0\}$ coincides with the unstable manifold $M^{u}$ of the autonomous system (2.2) with $l=l_{u}$ and $g_{l_{u}}(x, t) \equiv g_{l_{u}}^{-\infty}(x)$.

Similarly, we add to (2.2) the variable $\zeta=e^{-\varpi t}$ and we get

$$
\left(\begin{array}{c}
\dot{x}_{l_{s}} \\
\dot{y}_{l_{s}} \\
\dot{\zeta}
\end{array}\right)=\left(\begin{array}{ccc}
\alpha_{l_{s}} & 1 & 0 \\
0 & \gamma l_{s} & 0 \\
0 & 0 & -\varpi
\end{array}\right)\left(\begin{array}{c}
x_{l_{s}} \\
y_{l_{s}} \\
\zeta
\end{array}\right)+-g_{l_{s}}\left(x_{l_{s}},-\frac{\ln (\zeta)}{\omega}\right) .
$$

Since all the trajectories of (2.7) converge to the $\zeta=0$ plane as $t \rightarrow+\infty$, (2.7) will provide information on the asymptotic behavior of trajectories in the future. When $\boldsymbol{G}_{\boldsymbol{S}}$ holds, the origin admits a two-dimensional stable manifold denoted by $\boldsymbol{W}^{\boldsymbol{s}}$. For any $\tau \in \mathbb{R}, \tilde{W}_{l_{s}}^{s}(\tau)=\boldsymbol{W}^{s} \cap\left\{\zeta=e^{-\varpi \tau}\right\}$ is a one-dimensional manifold. Observe that $\tilde{W}_{l_{s}}^{s}(+\infty):=W^{s} \cap\{\zeta=0\}$ coincides with the stable manifold $M^{s}$ of the autonomous system (2.2) with $l=l_{s}$ and $g_{l_{s}}(x, t) \equiv g_{l_{s}}^{+\infty}(x)$.

Let $W_{l_{u}}^{u}(\tau)$ and $W_{l_{s}}^{s}(\tau)$ be such that $\tilde{W}_{l_{u}}^{u}(\tau)=W_{l_{u}}^{u}(\tau) \times\{z(\tau)\}$ and $\tilde{W}_{l_{s}}^{s}(\tau)=W_{l_{s}}^{s}(\tau) \times$ $\{\zeta(\tau)\}$.

Since $g(0, t)=0$ by assumption, the $z$-axis $(0,0, z)$ belongs to both $\boldsymbol{W}^{\boldsymbol{u}}$ and $\boldsymbol{W}^{\boldsymbol{s}}$.

\begin{tabular}{|l|l|l|l|l|l|}
\hline 220 & $\mathbf{2 5 4 6} \mathbf{3}$ & $\mathbf{B}$ & $\begin{array}{l}\text { Dispatch: 28/12/2015 } \\
\text { Total pages: 27 } \\
\text { Disk Received } \\
\text { Disk Used } \square\end{array}$ & $\begin{array}{l}\text { Journal: Commun. Math. Phys. } \\
\text { Not Used } \square \\
\text { Corrupted } \square \\
\text { Mismatch } \square\end{array}$ \\
\hline Jour. No & Ms. No.
\end{tabular}


Remark 2.6. We remark that $W_{l_{u}}^{u}(T)$ (respectively $W_{l_{s}}^{s}(T)$ ) depends continuously on $T \in$ $[-\infty,+\infty)$ (respectively on $T \in(-\infty,+\infty]$ ), see [23,26]. Indeed, if $W_{l_{u}}^{u}(T)$ (respectively $W_{l_{s}}^{s}(T)$ ) intersects transversally a line $L$ in a point $Q(T)$ for $T \in[-\infty,+\infty)$ (respectively for $T \in(-\infty,+\infty])$, then there is a neighbourhood $I$ of $T$ such that $W_{l_{u}}^{u}(\tau)$ (respectively $\left.W_{l_{s}}^{s}(\tau)\right)$ intersects $L$ in a point $\boldsymbol{Q}(\tau)$ for any $\tau \in I$, and $\boldsymbol{Q}(\tau)$ is continuous, see [26].

Remark 2.1 admits an extension to the non-autonomous case. From standard argument in invariant manifold theory, we know that in the origin $\boldsymbol{W}^{\boldsymbol{u}}$ is tangent to the plane $y=0$, while $\boldsymbol{W}^{s}$ is tangent to the plane $y=-(n-2) x$. However, we can get more with a construction involving exponential dichotomy, developed in [23], see also [14]. Denote by $\boldsymbol{x}_{l}(t ; \tau, \boldsymbol{Q})=\left(x_{l}(t ; \tau, \boldsymbol{Q}), y_{l}(t ; \tau, \boldsymbol{Q})\right)$ the trajectory of (2.2) satisfying the initial condition $\boldsymbol{x}_{l}(\tau)=Q \in \mathbb{R}^{2}$.

Lemma 2.7. Assume $\boldsymbol{G}_{\boldsymbol{u}}$ and $\boldsymbol{G}_{\boldsymbol{s}}$, then $W_{l_{u}}^{u}(\tau)$ is tangent to the line $y=0$, while $W_{l_{s}}^{s}(\tau)$ is tangent to the line $y=-(n-2) x$, for any $\tau \in \mathbb{R}$.

Proof. Assume $\boldsymbol{G}_{\boldsymbol{u}}$ and $\boldsymbol{G}_{\boldsymbol{s}}$, and set

$$
\begin{aligned}
& w^{u}(\tau):=\left\{\boldsymbol{Q} \mid \lim _{t \rightarrow-\infty} \boldsymbol{x}_{l_{u}}(t ; \tau, \boldsymbol{Q})=(0,0)\right\}, \\
& w^{s}(\tau):=\left\{\boldsymbol{Q} \mid \lim _{t \rightarrow \infty} \boldsymbol{x}_{l_{s}}(t ; \tau, \boldsymbol{Q})=(0,0)\right\} .
\end{aligned}
$$

It can be proved that $w^{u}(\tau)$ and $w^{s}(\tau)$ are one-dimensional manifolds, since $g_{l_{u}}(x, t)$ and $g_{l_{s}}(x, t)$ are uniformly continuous for $t \leq \tau$ and for $t \geq \tau$, respectively, see [23,24]. In fact, from $\boldsymbol{G}_{\boldsymbol{u}}$ and $\boldsymbol{G}_{\boldsymbol{S}}$ we deduce that the manifold $W_{l_{u}}^{u}(\tau)$ coincides with the manifold $w^{u}(\tau)$ defined in (2.8), and $W_{l_{s}}^{s}(\tau)$ coincides with $w^{s}(\tau)$, for any $\tau \in \mathbb{R}$. Moreover, from $\boldsymbol{G}_{\mathbf{1}}$ we know that $g_{l_{u}}(x, t)=o(x)$ uniformly for $t \leq 0$, and $g_{l_{s}}(x, t)=o(x)$ uniformly for $t \geq 0$, thus $w^{u}(\tau)$ is tangent to the line $y=0$, while $w^{s}(\tau)$ is tangent to the line $y=-(n-2) x$, for any $\tau \in \mathbb{R}$. Hence, the thesis follows.

In order to understand the mutual position of $\boldsymbol{W}^{\boldsymbol{u}}$ and $\boldsymbol{W}^{\boldsymbol{s}}$ at a fixed instant $\tau$, we introduce the manifolds:

$$
\begin{aligned}
& W_{l_{s}}^{u}(\tau):=\left\{\boldsymbol{R}:=\boldsymbol{Q} e^{-\left(\alpha_{l_{u}}-\alpha_{l_{s}}\right) \tau} \in \mathbb{R}^{2} \mid \boldsymbol{Q} \in W_{l_{u}}^{u}(\tau)\right\}, \\
& W_{l_{u}}^{s}(\tau):=\left\{\boldsymbol{Q}:=\boldsymbol{R} e^{\left(\alpha_{l_{u}}-\alpha_{l_{s}}\right) \tau} \in \mathbb{R}^{2} \mid \boldsymbol{R} \in W_{l_{s}}^{s}(\tau)\right\} .
\end{aligned}
$$

As in the autonomous case, the origin splits $W_{l}^{u}(\tau)$ (and $W_{l}^{s}(\tau)$ ) in two components, say $W_{l}^{u,+}(\tau)$ which leaves the origin and enters $x>0$ (respectively $W_{l}^{s,+}(\tau)$ ), and $W_{l}^{u,-}(\tau)$ which leaves the origin and enters $x<0$ (resp. $\left.W_{l}^{s,-}(\tau)\right)$, for $l=l_{u}, l_{s}$. Similarly, we denote by $\boldsymbol{W}^{\boldsymbol{u},+}$ and $\boldsymbol{W}^{\boldsymbol{u},-}$ (respectively $\boldsymbol{W}^{\boldsymbol{s},+}$ and $\boldsymbol{W}^{\boldsymbol{s},-}$ ) the two components in which the $z$-axis divides $\boldsymbol{W}^{\boldsymbol{u}}$ (resp. $\boldsymbol{W}^{\boldsymbol{s}}$ ). From [14,17,18], we are able to extend Remark 2.2 to the non-autonomous case:

Lemma 2.8. Consider the trajectory $\boldsymbol{x}_{l_{u}}(t ; \tau, \boldsymbol{Q})$ of (2.2) with $l=l_{u}$ and the corresponding trajectory $\boldsymbol{x}_{l_{s}}(t ; \tau, \boldsymbol{R})$ of $(2.2)$ with $l=l_{s}$. Then, $\boldsymbol{R}=\boldsymbol{Q} e^{-\left(\alpha_{l_{u}}-\alpha_{l_{s}}\right) \tau}$. Let $u(r)$ be the corresponding solution of (1.2). Assume $\boldsymbol{G}_{\boldsymbol{u}}$ and $\boldsymbol{G}_{\boldsymbol{s}}$, then

$$
\begin{aligned}
& u(r) \text { is a regular solution } \Longleftrightarrow \boldsymbol{Q} \in W_{l_{u}}^{u}(\tau) \text { or } \boldsymbol{R} \in W_{l_{s}}^{u}(\tau), \\
& u(r) \text { is a fast decay solution } \Longleftrightarrow \boldsymbol{R} \in W_{l_{s}}^{s}(\tau) \text { or } \boldsymbol{Q} \in W_{l_{u}}^{s}(\tau) .
\end{aligned}
$$

Moreover, $u(0)=d>0$ iff $\boldsymbol{Q} \in W_{l_{u}}^{u,+}(\tau)$, and $d<0$ iff $\boldsymbol{Q} \in W_{l_{u}}^{u,-}(\tau) ; \lim _{r \rightarrow \infty} u(r) r^{n-2}$ $=L>0$ iff $\boldsymbol{R} \in W_{l_{s}}^{s,+}(\tau)$, and $L<0$ iff $\boldsymbol{R} \in W_{l_{s}}^{s,-}(\tau)$.

\begin{tabular}{|l|l|l|l|l|l|}
\hline 220 & $\mathbf{2 5 4 6}$ & $\mathbf{B}$ & $\begin{array}{l}\text { Dispatch: 28/12/2015 } \\
\text { Total pages: 27 } \\
\text { Disk Received } \\
\text { Disk Used } \square\end{array}$ & $\begin{array}{l}\text { Journal: Commun. Math. Phys. } \\
\text { Not Used } \square \\
\text { Corrupted } \square \\
\text { Mismatch } \square\end{array}$ \\
\hline
\end{tabular}


We complete the discussion of the correspondences between (1.2) and (2.2) with the analysis of singular and slow decay solutions, based on standard invariant manifold theory. For analogous considerations, we refer, among others, to [18]. Assume $\boldsymbol{G}_{\boldsymbol{u}}$ with $l_{u}>2_{*}$, and denote by $\boldsymbol{P}^{ \pm}(-\infty)=\left(P_{x}^{ \pm}(-\infty),-\alpha_{l_{u}} P_{x}^{ \pm}(-\infty)\right)$ the critical points (different from the origin) of the autonomous system (2.2), where $l=l_{u}$ and $g_{l_{u}}(x, t) \equiv$ $g_{l_{u}}^{-\infty}(x)$. Then, observe that $\left(\boldsymbol{P}^{ \pm}(-\infty), 0\right)$ are critical points of (2.6), and they admit an unstable manifold which is one-dimensional for $l_{u} \geq 2^{*}$ and two-dimensional for $2_{*}<l_{u}<2^{*}$. If $\left(\boldsymbol{Q}, e^{\varpi \tau}\right)$ belongs to such a manifold, then $\lim _{t \rightarrow-\infty} \boldsymbol{x}_{l_{u}}(t ; \tau, \boldsymbol{Q})=$ $\boldsymbol{P}^{ \pm}(-\infty)$, and, consequently, the corresponding solution $u(r)$ of (1.2) is a singular solution satisfying $\lim _{r \rightarrow 0} u(r) r^{\alpha_{l}}=P_{x}^{ \pm}(-\infty)$.

Similarly, assume $\boldsymbol{G}_{\boldsymbol{s}}$, and denote by $\boldsymbol{P}^{ \pm}(+\infty)=\left(P_{x}^{ \pm}(+\infty),-\alpha_{l_{s}} P_{x}^{ \pm}(+\infty)\right)$ the critical points of the autonomous system (2.2), where $l=l_{s}$ and $g_{l_{s}}(x, t) \equiv g_{l_{s}}^{+\infty}(x)$. Then, observe that $\left(\boldsymbol{P}^{ \pm}(+\infty), 0\right)$ are critical points of $(2.7)$, and they admit a stable manifold which is one-dimensional for $2_{*}<l_{s} \leq 2^{*}$ and two-dimensional for $l_{s}>2^{*}$. If $\left(\boldsymbol{Q}, e^{-\varpi \tau}\right)$ belongs to such a manifold, then $\lim _{t \rightarrow \infty} \boldsymbol{x}_{l_{s}}(t ; \tau, \boldsymbol{Q})=\boldsymbol{P}^{ \pm}(+\infty)$, and, consequently, the corresponding solution $u(r)$ of (1.2) is a slow decay solution satisfying $\lim _{r \rightarrow \infty} u(r) r^{\alpha_{l_{s}}}=P_{x}^{ \pm}(+\infty)$.

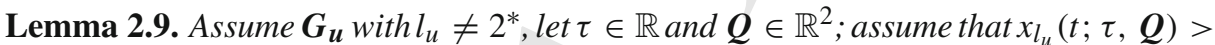
0 for any $t \leq \tau$, and let $u(r)$ be the corresponding solution of (1.2). Then, either $\boldsymbol{Q} \in W_{l_{u}}^{u,+}(\tau)$ or $\lim _{t \rightarrow-\infty} \boldsymbol{x}_{l_{u}}(t ; \tau, \boldsymbol{Q})=\boldsymbol{P}^{+}(-\infty)$; in the former case $u(r)$ is regular and $u(0)>0$, in the latter it is singular and $\lim _{r \rightarrow 0} u(r) r^{\alpha_{l_{u}}}=P_{x}^{+}(-\infty)$.

If $l_{u}=2^{*}$ we have a third possibility: $\boldsymbol{x}_{l_{u}}(t ; \tau, \boldsymbol{Q})$ may be uniformly positive and bounded, so $u(r)$ is singular.

Similarly, assume $\boldsymbol{G}_{\boldsymbol{S}}$ with $l_{s} \neq 2^{*}$, let $\tau \in \mathbb{R}$ and $\boldsymbol{Q} \in \mathbb{R}^{2}$; assume that $x_{l_{s}}(t ; \tau, \boldsymbol{Q})>$ 0 for any $t \geq \tau$; let $u(r)$ be the corresponding solution of (1.2). Then, either $Q \in$ $W_{l_{s}}^{s,+}(\tau)$, or $\lim _{t \rightarrow \infty} \boldsymbol{x}_{l_{s}}(t ; \tau, \boldsymbol{Q})=\boldsymbol{P}^{+}(+\infty)$; in the former case $u(r)$ has fast decay with $\lim _{r \rightarrow \infty} u(r) r^{n-2}=L>0$, in the latter it has slow decay with $\lim _{r \rightarrow \infty} u(r) r^{\alpha l_{s}}=$ $P_{x}^{+}(+\infty)$.

If $l_{s}=2^{*}$ we have a third possibility: $\boldsymbol{x}_{l_{s}}(t ; \tau, \boldsymbol{Q})$ may be uniformly positive and bounded, so u(r) has slow decay.

We emphasize that the symmetric result for definitely negative solutions holds true; the corresponding statement will be omitted for brevity.

Hence, under the assumptions of Theorem 2.4, $u$ is either regular, or singular, or it has infinitely many zeroes for $r<1$; moreover, it has either fast or slow decay, or it has infinitely many zeroes for $r>1$.

We introduce a further Lemma to clarify the relationship between regular solutions $u(r, d)$ of (1.2) and the corresponding trajectories $\boldsymbol{x}_{l_{u}}(t ; \tau, \boldsymbol{Q})$ of (2.2). The automonous case can be easily treated thanks to invariance for translations in $t$. In particular, fix $Q \in$ $M^{u,+}$ and consider the trajectory $\boldsymbol{x}_{l_{u}}(t ; \tau, \boldsymbol{Q})$ of (2.2) and the corresponding solution $u(r, d(\tau))$ of (1.2). Then, arguing as in the proof of Remark 1.3, we find that $d(\tau)=$ $d(0) e^{-\alpha_{l_{u}} \tau}$, from which it follows that $d$ is a strictly decreasing, continuous function of $\tau$ with $\lim _{\tau \rightarrow-\infty} d(\tau)=+\infty$ and $\lim _{\tau \rightarrow+\infty} d(\tau)=0$. In the non-autonomous case, an analogous property is satisfied.

Lemma 2.10. Assume $\boldsymbol{G}_{\boldsymbol{u}}$ with $l_{u}>2_{*}$, fix $T \in \mathbb{R}$, and let $\Upsilon_{u}(\cdot, T):[0,+\infty) \rightarrow$ $W_{l_{u}}^{u,+}(T)$ be a smooth (bijective) parametrization of $W_{l_{u}}^{u,+}(T)$ such that $\Upsilon_{u}(0, T)=$ $(0,0)$. Let $u(r, d(U))$ be the solution of (1.2) corresponding to $\boldsymbol{x}_{l_{u}}\left(t ; T, \Upsilon_{u}(U, T)\right)$.

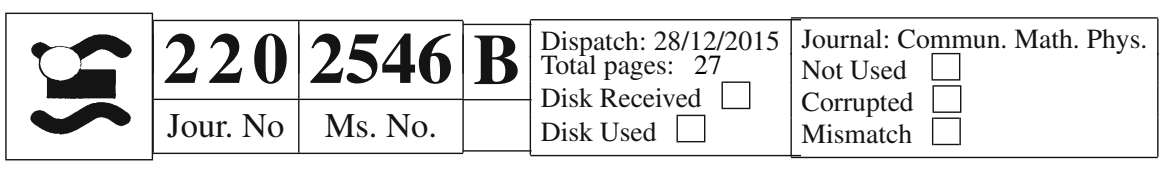


Then, $d(U)$ is a strictly increasing function such that $d(0)=0$ and $\lim _{U \rightarrow+\infty} d(U)=$ $+\infty$.

Note that we can parametrize $W_{l_{u}}^{u,+}(T)$ directly with $d$. An analogous statement can be written for $W_{l_{s}}^{s,+}(T)$ (which can be parametrized by $L:=\lim _{r \rightarrow \infty} u(r) r^{n-2}$ ).

Proof. Consider the parametrization of $W_{l_{u}}^{u,+}(T)$ given by $\Upsilon_{u}(\cdot, T):[0,+\infty) \rightarrow$ $W_{l_{u}}^{u,+}(T)$ such that $\Upsilon_{u}(0, T)=(0,0)$. Observe first that, starting from $\Upsilon_{u}(\cdot, T)$, we can construct a parametrization of $W_{l_{u}}^{u,+}(\tau)$ for any $\tau \in \mathbb{R}$, by setting $\Upsilon_{u}(U, \tau):=$ $\boldsymbol{x}_{l_{u}}\left(\tau ; T, \Upsilon_{u}(U, T)\right)$. In fact, the function $\Upsilon_{u}:[0,+\infty) \times \mathbb{R} \rightarrow \mathbb{R}^{2}$ is continuous in both the variables, and $(U, \tau) \rightarrow\left(\Upsilon_{u}(U, \tau), z(\tau)\right)$ is an injective map in $\boldsymbol{W}^{\boldsymbol{u},+}$. According to this parametrization, $\boldsymbol{x}_{l_{u}}\left(t ; \tau, \Upsilon_{u}(U, \tau)\right)$ coincides with $\boldsymbol{x}_{l_{u}}\left(t ; T, \Upsilon_{u}(U, T)\right)$ and corresponds to the given solution $u(r, d(U))$ for any $\tau \in \mathbb{R}$. Note, however, that this parametrization cannot be extended to a continuous parametrization of the whole $\boldsymbol{W}^{\boldsymbol{u},+}$, since $\Upsilon_{u}(U, \tau) \rightarrow(0,0)$ as $\tau \rightarrow-\infty$, which does not provide a parametrization of $W_{l_{u}}^{u,+}(-\infty)$.

Let $B(\delta)$ be the closed ball of radius $\delta>0$ centered in the origin. We can find a (small) $\delta>0$, independent of $\tau$, such that the connected component $W_{l_{u}, \text { loc }}^{u,+}(\tau)$ of $W_{l_{u}}^{u,+}(\tau) \cap B(\delta)$ containing the origin is a graph on its tangent space, i.e. the $x$-axis, for any $\tau \leq 0$, see e.g. [24,26]. Moreover, for any $\bar{U}>0$, we can find a large enough $N(\bar{U})>0$ such that $\Upsilon_{u}(U, \tau) \in W_{l_{u}, \text { loc }}^{u,+}(\tau)$, whenever $0 \leq U \leq \bar{U}$ and $\tau \leq-N(\bar{U})$.

We now show that $d(U)$ is strictly increasing; the other properties easily follow. Let $U_{2}>U_{1}$, then $\Upsilon_{u}\left(U_{i}, \tau\right) \in W_{l_{u}, \text { loc }}^{u,+}(\tau)$ for any $\tau \leq-N\left(U_{2}\right)$ and for $i=1,2$. Hence, $\Upsilon(\cdot, \tau):\left[0, U_{2}\right] \rightarrow W_{l_{u}}^{u,+}(\tau)$ is a graph on the $x$-axis, for any $\tau<-N\left(U_{2}\right)$. In particular, $x_{l_{u}}\left(\tau ; T, \Upsilon\left(U_{1}, T\right)\right)<x_{l_{u}}\left(\tau ; T, \Upsilon\left(U_{2}, T\right)\right)$ for any $\tau<-N\left(U_{2}\right)$, and, consequently, $u\left(r, d\left(U_{1}\right)\right)<u\left(r, d\left(U_{2}\right)\right)$ for any $r<e^{-N\left(U_{2}\right)}$. Thus, $d\left(U_{1}\right)<d\left(U_{2}\right)$.

2.1. Kelvin inversion. An important tool in the analysis of Eq. (1.2) is a change of variables classically known as Kelvin inversion, useful to transfer the information on regular and singular solutions to fast and slow decay solutions. Set

$$
s=r^{-1}, \quad \tilde{u}(s)=s^{2-n} u(1 / s), \quad \tilde{f}(\tilde{u}, s)=f\left(\tilde{u} s^{n-2}, 1 / s\right) s^{-2-n} .
$$

From a straightforward computation, we see that $u(r)$ satisfies (1.2) if and only if $\tilde{u}(s)$ satisfies the following equation

$$
\frac{d}{d s}\left[\tilde{u}_{s}(s) s^{n-1}\right]+\tilde{f}(\tilde{u}(s), s) s^{n-1}=0,
$$

where $\tilde{u}_{s}:=\frac{d \tilde{u}}{d s}$. The change of variables (2.10) brings regular, singular, fast decay and slow decay solutions of (1.2) into respectively fast decay, slow decay, regular and singular solutions $\tilde{u}(s)$ of (2.11), and viceversa. In [18], it has been recently observed that clearer and more detailed information can be acquired by combining (2.10) with (2.1). Hence, when $f$ satisfies $\boldsymbol{G}_{\boldsymbol{u}}$ with $l=l_{u}>2_{*}$, then $\tilde{f}$ satisfies $\boldsymbol{G}_{\boldsymbol{s}}$ with $l=L_{s}>2_{*}$, where

$$
L_{s}=2-\frac{2}{\gamma l_{u}}=\frac{2\left[l_{u}(n-1)-2 n\right]}{l_{u}(n-2)-2 n+2}, \quad \alpha_{L_{s}}=-\gamma_{l_{u}}, \quad \gamma_{L_{s}}=-\alpha_{l_{u}} .
$$

\begin{tabular}{|l|l|l|l|l|l|}
\hline 220 & 2546 & $\mathbf{B}$ & $\begin{array}{l}\text { Dispatch: 28/12/2015 } \\
\text { Total pages: 27 } \\
\text { Disk Received } \square \\
\text { Disk Used } \square\end{array}$ & $\begin{array}{l}\text { Journal: Commun. Math. Phys. } \\
\text { Not Used } \square \\
\text { Corrupted } \square \\
\text { Mismatch } \square\end{array}$ \\
\hline
\end{tabular}


Analogously, when $f$ satisfies $\boldsymbol{G}_{\boldsymbol{s}}$ with $l=l_{s}>2_{*}$, then $\tilde{f}$ satisfies $\boldsymbol{G}_{\boldsymbol{u}}$ with $l=L_{u}>$ $2_{*}$, where

$$
L_{u}=2-\frac{2}{\gamma_{l_{s}}}=\frac{2\left[l_{s}(n-1)-2 n\right]}{l_{s}(n-2)-2 n+2}, \quad \alpha_{L_{u}}=-\gamma_{l_{s}}, \quad \gamma_{L_{u}}=-\alpha_{l_{s}} .
$$

Setting

$$
L=L_{l}=2-\frac{2}{\gamma_{l}}, \quad \tilde{x}(\tilde{t})=\tilde{u}(s) s^{\alpha_{L}}, \quad s=\mathrm{e}^{\tilde{t}},
$$

the Kelvin inversion transforms system (2.2) into the following

$$
\left(\begin{array}{c}
\frac{d \tilde{x}}{d \tilde{t}} \\
\frac{d \tilde{y}}{d \tilde{t}}
\end{array}\right)=\left(\begin{array}{cc}
-\gamma_{l} & 1 \\
0 & -\alpha_{l}
\end{array}\right)\left(\begin{array}{l}
\tilde{x} \\
\tilde{y}
\end{array}\right)+\left(\begin{array}{c}
0 \\
-g_{l}(\tilde{x},-\tilde{t})
\end{array}\right) .
$$

Note that to pass from (2.2) to (2.12) we just need to replace $\alpha$ by $-\gamma, \gamma$ by $-\alpha$ and $g_{l}(x, t)$ by $g_{l}(x,-t)$. This way it is more clear that, roughly speaking, the difference between (1.2) and (2.11) consists in a simple reversion of time. Provided that we choose $l>2_{*}$, observe that $L>2^{*} \Longleftrightarrow l<2^{*}$ and $L<2^{*} \Longleftrightarrow l>2^{*}$. In particular, subcritical systems are driven in supercritical systems, and viceversa. Furthermore, $W_{l_{s}}^{s}(T)$ is brought into $W_{L_{u}}^{u}(-T)$, and $W_{l_{u}}^{u}(T)$ is brought into $W_{L_{s}}^{s}(-T)$. This will help us to automatically translate results for regular and singular solutions into results for fast and slow decay solutions, and viceversa.

\section{The Main Result}

In the whole section we assume the hypotheses of Theorem 2.4 without further mentioning.

From $\boldsymbol{G}_{\boldsymbol{S}}$ we know that $W_{l_{s}}^{s}(T)$ exists for any $T \in \mathbb{R}$. We recall that $W_{l_{s}}^{s}(+\infty)$ coincides with the stable manifold $M^{s}$ of the autonomous system (2.2) with $l=l_{s}$ and $g_{l_{s}}(x, t) \equiv g_{l_{s}}^{+\infty}(x)$. Since $l_{s}>2^{*}$ by assumption $(2.5), W_{l_{s}}^{s,+}(+\infty)$ and $W_{l_{s}}^{s,-}(+\infty)$ are unbounded spirals which rotate intersecting transversally the coordinate axes infinitely many times, see e.g. $[13,14,17]$ and Fig. 1 . Note that these intersections are unbounded sequences which do not accumulate in any point.

For every solution $\boldsymbol{x}_{l}:=\left(x_{l}, y_{l}\right)$ of (2.2), we introduce polar coordinates

$$
\theta_{l}=\arctan \left(y_{l} / x_{l}\right), \quad \rho_{l}=\left\|\boldsymbol{x}_{l}\right\| .
$$

Taking into account (2.1), we stress that if we switch between different values of $l$, say $l$ and $L$, we get $\rho_{L}(t)=\exp \left[\left(\alpha_{L}-\alpha_{l}\right) t\right] \rho_{l}(t)$ and $\theta_{L}(t)=\theta_{l}(t)$, so we drop the subscript in $\theta$.

From (2.1) and (2.2), we easily obtain

$$
\frac{d \theta}{d t}=(2-n) \sin \theta \cos \theta-\sin ^{2} \theta-\frac{g_{l}\left(\rho_{l} \cos \theta, t\right)}{\rho_{l}} \cos \theta .
$$

Thus, the flow of (2.2) on the coordinate axes is transversal, and rotates clockwise for any $t \in \mathbb{R}$.

Lemma 3.1. The integer part of $\left(\frac{2 \theta(t)}{\pi}\right)$ is decreasing in $t$.

\begin{tabular}{|l|l|l|l|l|l|}
\hline 220 & 2546 & $\mathbf{B}$ & $\begin{array}{l}\text { Dispatch: 28/12/2015 } \\
\text { Total pages: 27 } \\
\text { Disk Received } \\
\text { Disk Used } \square\end{array}$ & $\begin{array}{l}\text { Journal: Commun. Math. Phys. } \\
\text { Not Used } \square \\
\text { Corrupted } \square \\
\text { Mismatch } \square\end{array}$ \\
\hline
\end{tabular}


From (3.2), according to Remark 2.1 and Lemma 2.8, we deduce that

Lemma 3.2. Let $\left(\boldsymbol{x}_{l_{s}}(t), \zeta(t)\right)$ and $\left(\overline{\boldsymbol{x}}_{l_{s}}(t), \zeta(t)\right)$ be trajectories in $\boldsymbol{W}^{\boldsymbol{s},+}$ and $\boldsymbol{W}^{\boldsymbol{s},-}$, respectively; let $\theta^{s,+}(t)$ and $\theta^{s,-}(t)$ be the angular coordinates associated with $\boldsymbol{x}_{l_{s}}(t)$ and $\overline{\boldsymbol{x}}_{l_{s}}(t)$. Then, $\lim _{t \rightarrow+\infty} \theta^{s,+}(t)=\bar{\theta}:=-\arctan (n-2) \in\left(-\frac{\pi}{2}, 0\right)$, and $\lim _{t \rightarrow+\infty} \theta^{s,-}(t)$ $=\bar{\theta}-\pi$.

For any $\tau \in \mathbb{R}$ we construct a continuous parametrizations of $W_{l_{s}}^{s, \pm}(\tau)$, by setting $\Sigma_{l_{s}}^{s, \pm}(\cdot, \zeta(\tau)):[0,+\infty) \rightarrow W_{l_{s}}^{s, \pm}(\tau) \times\{\zeta(\tau)\}$ such that $\Sigma_{l_{s}}^{s, \pm}(0, \zeta(\tau))=(0,0, \zeta(\tau))$. Then, we define continuous parametrizations of $W_{l_{s}}^{s, \pm}(+\infty)$, by setting $\Sigma_{l_{s}}^{s, \pm}(\cdot, 0)$ : $[0,+\infty) \rightarrow W_{l_{s}}^{s, \pm}(+\infty) \times\{0\}$ such that $\Sigma_{l_{s}}^{s, \pm}(0,0)=(0,0,0)$. We have in fact obtained two parameters bijective parametrizations $\Sigma_{l_{s}}^{s, \pm}:[0,+\infty) \times[0,+\infty) \rightarrow \boldsymbol{W}^{s, \pm}$ such that $\Sigma_{l_{s}}^{s, \pm}(0, \zeta)=(0,0, \zeta)$, which may be assumed to be continuous in both the variables, in view of Remark 2.6.

Now we fix $T \in \mathbb{R}$ and we choose points $Q^{ \pm}(T) \in W_{l_{s}}^{s, \pm}(T)$; denote by $\bar{W}_{l_{s}}^{s, \pm}(T)$ the branches of $W_{l_{s}}^{s, \pm}(T)$ between the origin and $Q^{ \pm}(T)$; let $S_{T}^{ \pm}$be the positive numbers satisfying $\Sigma_{l_{s}, \pm}\left(S_{T}^{ \pm}, \zeta(T)\right)=\left(Q^{ \pm}(T), \zeta(T)\right)$.

By adopting the same arguments in $[2,18,27]$, it is possible to show that the number of rotations around the origin realized by the flow $\boldsymbol{x}_{l_{s}}\left(\cdot ; T, Q^{ \pm}(T)\right)$ in the interval of time $[T,+\infty)$ coincides with the number of rotations performed by the branch $\bar{W}_{l_{s}}^{s, \pm}(T)$.

For this purpose, let us introduce the parametrization in polar coordinates of $\tilde{W}_{l_{s}}^{s, \pm}(T)=W_{l_{s}}^{s, \pm}(T) \times\{\zeta(T)\}$, by

$$
\Sigma_{l_{s}}^{s, \pm}(S, \zeta)=\left(R_{l_{s}}^{s, \pm}(S, \zeta) \cos \left(\phi^{s, \pm}(S, \zeta)\right), R_{l_{s}}^{s, \pm}(S, \zeta) \sin \left(\phi^{s, \pm}(S, \zeta)\right), \zeta\right),
$$

where $\zeta=\zeta(T)=e^{-\varpi T}$.

According to (3.1), the trajectories $\boldsymbol{x}_{l_{s}}\left(t ; T, Q^{ \pm}(T)\right)$ can be parametrized by

$$
\begin{aligned}
& \boldsymbol{x}_{l_{s}}\left(t ; T, \boldsymbol{Q}^{+}(T)\right)=\left(\rho_{l_{s}}^{s,+}(t) \cos \left(\theta^{s,+}(t)\right), \rho_{l_{s}}^{s,+}(t) \sin \left(\theta^{s,+}(t)\right)\right), \\
& \boldsymbol{x}_{l_{s}}\left(t ; T, \boldsymbol{Q}^{-}(T)\right)=\left(\rho_{l_{s}}^{s,-}(t) \cos \left(\theta^{s,-}(t)\right), \rho_{l_{s}}^{s,-}(t) \sin \left(\theta^{s,-}(t)\right)\right) .
\end{aligned}
$$

Following $[2,18,27]$, given a curve $\boldsymbol{\gamma}:[a, b] \rightarrow \mathbb{R}^{2}$, we define its rotation number $w(\boldsymbol{\gamma})$ by setting

$$
w(\boldsymbol{\gamma}):=\left[\frac{\theta_{\boldsymbol{\gamma}}(b)-\theta_{\boldsymbol{\gamma}}(a)}{2 \pi}\right],
$$

where [.] denotes the integer part and $\boldsymbol{\gamma}(t)=\left(\rho_{\boldsymbol{\gamma}}(t) \cos \theta_{\boldsymbol{\gamma}}(t), \rho_{\boldsymbol{\gamma}}(t) \sin \theta_{\boldsymbol{\gamma}}(t)\right)$. As pointed out in [18], we can extend this definition to a curve $\gamma$ defined in a semi-open interval $[a, b)$ if $\lim _{t \rightarrow b^{-}} \theta_{\gamma}(t)$ exists (even if it is infinite). So, we can extend the definition to a curve $\gamma(t)$ defined on $[a,+\infty)$ converging to $(0,0)$ as $t \rightarrow+\infty$, provided that $\gamma(t) \neq(0,0)$ for any $t \in[a,+\infty)$ and $\lim _{t \rightarrow+\infty} \theta_{\gamma}(t)$ exists. By adapting the argument of [2,27], and, in particular, of Sect. 4 in [18] we can show the following

Lemma 3.3. $[2,18,27]$. Take $T \in \mathbb{R}, Q^{+}(T) \in W_{l_{s}}^{s,+}(T)$ and $Q^{-}(T) \in W_{l_{s}}^{s,-}(T)$, then

$$
\begin{gathered}
w\left(\Sigma_{l_{s}}^{s,+}(\cdot, \zeta(T))\right)=-w\left(\boldsymbol{x}_{l_{s}}\left(\cdot ; T, Q^{+}(T)\right)\right), \\
w\left(\Sigma_{l_{s}}^{s,-}(\cdot, \zeta(T))\right)=-w\left(\boldsymbol{x}_{l_{s}}\left(\cdot ; T, Q^{-}(T)\right)\right),
\end{gathered}
$$

where $\Sigma_{l_{s}}^{s, \pm}$ and $\boldsymbol{x}_{l_{s}}$ are restricted to the intervals $\left[0, S_{T}^{ \pm}\right]$and $[T,+\infty)$, resp.

\begin{tabular}{|l|l|l|l|l|l|l|}
\hline 220 & 2546 & $\mathbf{B}$ & $\begin{array}{l}\text { Dispatch: 28/12/2015 } \\
\text { Total pages: 27 } \\
\text { Disk Received } \square \\
\text { Disk Used } \square\end{array}$ & $\begin{array}{l}\text { Journal: Commun. Math. Phys. } \\
\text { Not Used } \square \\
\text { Corrupted } \square \\
\text { Mismatch } \square\end{array}$ \\
\hline
\end{tabular}


Sketch of the proof We just sketch the proof, referring to [2,18,27] for details. We discuss the first equality in (3.6). The second one is analogous.

Let $\Gamma^{a}$ be a path with the same graph and orientation as the curve $\left(\boldsymbol{x}_{l_{s}}\left(t ; T, Q^{+}(T)\right)\right.$, $\zeta(t))$ defined for $t \geq T$; let $\Gamma^{b}$ be the path obtained following first $\bar{W}_{l_{s}}^{s,+}(T) \times\{\zeta(T)\}$ from $\left(Q^{+}(T), \zeta(T)\right)$ to $(0,0, \zeta(T))$ and then the segment which joins $(0,0, \zeta(T))$ to $(0,0,0)$. Note that the orthogonal projection of $\left(\boldsymbol{x}_{l_{s}}\left(t ; T, Q^{+}(T)\right), \zeta(t)\right)$ on $\mathbb{R}^{2} \times$ $\{\zeta(T)\}$ does not coincide with $\bar{W}_{l_{s}}^{s,+}(T) \times\{\zeta(T)\}$. Nevertheless, by adapting the argument in [18, Sect. 4], we can construct an homotopy between $\Gamma^{a}$ and $\Gamma^{b}$ which preserves the endpoints $\left(Q^{+}(T), \zeta(T)\right)$ and $(0,0,0)$. This homotopy is obtained projecting $\left(x_{l_{s}}\left(t ; T, Q^{+}(T)\right), \zeta(t)\right)$ on $\mathbb{R}^{2} \times\{\zeta(T)\}$ not orthogonally, but following $\boldsymbol{W}^{s,+}$. Once we build the homotopy, from a topological argument we deduce that the rotation numbers of $\Gamma^{a}$ and $\Gamma^{b}$ are equal, see [18, Sect. 4], and [2,27]. The minus sign in (3.6) follows from the fact that $\Gamma^{b}$ has opposite orientation with respect to $\Sigma_{l_{s}}^{s,+}(\cdot, \zeta(T))$.

Proposition 3.4. Take $T \in \mathbb{R}, Q^{+}(T) \in W_{l_{s}}^{s,+}(T), Q^{-}(T) \in W_{l_{s}}^{s,-}(T)$, let $S_{T}^{ \pm}$be the positive numbers satisfying $\Sigma_{l_{s}}^{s, \pm}\left(S_{T}^{ \pm}, \zeta(T)\right)=\left(Q^{ \pm}(T), \zeta(T)\right)$. Consider the parametrizations (3.3) in polar coordinates of $W_{l_{s}}^{s, \pm}(T)$, then, $\phi^{s, \pm}\left(S_{T}^{ \pm}, \zeta\right)=\theta^{s, \pm}(T)$. Moreover, $\boldsymbol{x}_{l_{s}}\left(\cdot ; T, Q^{+}(T)\right)$ and $\boldsymbol{x}_{l_{s}}\left(\cdot ; T, Q^{-}(T)\right)$ perform in the interval of time $[T,+\infty)$ the angles $\left(\bar{\theta}-\phi^{s,+}\left(S_{T}^{+}, \zeta\right)\right)$ and $\left(\bar{\theta}-\pi-\phi^{s,-}\left(S_{T}^{-}, \zeta\right)\right)$ around the origin, respectively.

Proof. By Lemmas 3.1 and 3.2, $\boldsymbol{x}_{l_{s}}\left(t ; T, Q^{+}(T)\right)$ performs in the interval of time $[T,+\infty)$ the angle $\left(\bar{\theta}-\theta^{s,+}(T)\right)$ around the origin. The thesis follows by using Lemma 3.3. The proof for $\boldsymbol{x}_{l_{s}}\left(t ; T, Q^{-}(T)\right)$ is analogous.

From $\boldsymbol{G}_{\boldsymbol{u}}, \boldsymbol{G}_{\boldsymbol{s}}$ with $2_{*}<l_{u}<2^{*}<l_{s}$, we deduce the following lemma.

Lemma 3.5. $W_{l_{s}}^{s,+}(T)$ and $W_{l_{s}}^{s,-}(T)$ are spirals rotating counterclockwise starting from $(0,0)$, and they intersect the coordinate axes infinitely many times for every $T \in \mathbb{R}$.

Proof. We develop the proof for $W_{l_{s}}^{s,+}(T)$; the case of $W_{l_{s}}^{s,-}(T)$ might be treated equivalently. As observed at the beginning of Sect. 3, we recall that the lemma holds for $M^{s,+}=W_{l_{s}}^{s,+}(+\infty)$. According to Remark 2.6, from a standard continuity argument we deduce that for every $k \in \mathbb{N} \backslash\{0\}$ there exists $T_{k}$ such that $W_{l_{s}}^{s,+}(T)$ intersects the $y$ coordinate axis at least $k$ times, for $T \geq T_{k}$. Let us denote by $\hat{W}_{l_{s}}^{s,+}\left(T_{k}\right)$ the branch of $W_{l_{s}}^{s,+}\left(T_{k}\right)$ between the origin and its $k$ th intersection with the $y$-axis, called $\boldsymbol{P}\left(T_{k}\right)$. According to Remark 2.5, the trajectory $\boldsymbol{x}_{l_{s}}\left(t ; T_{k}, \boldsymbol{P}\left(T_{k}\right)\right)$ of (2.2) can be continued for any $t<T_{k}$. Consider now $T<T_{k}$. Denote by $\hat{W}_{l_{s}}^{s,+}(T)$ the branch of $W_{l_{s}}^{s,+}(T)$ between the origin and $\boldsymbol{x}_{l_{s}}\left(T ; T_{k}, \boldsymbol{P}\left(T_{k}\right)\right)$, and by $N(T)$ the number of intersection of $\hat{W}_{l_{s}}^{s,+}(T)$ with the $y$-axis. Let $\theta^{s,+}(t)$ be the angular coordinate of $\boldsymbol{x}_{l_{s}}\left(t ; T_{k}, \boldsymbol{P}\left(T_{k}\right)\right)$.

Since the flow of (2.2) on the coordinate axes rotates clockwise (see Lemma 3.1), taking into account Proposition 3.4, we infer that $N(t)$ is decreasing with $t$ for any $t \leq T_{k}$, whence $N(T) \geq k$ for $T<T_{k}$. This completes the proof.

From Lemma 3.5, recalling notation (3.3), we see that $\lim _{S \rightarrow+\infty} \phi^{s, \pm}(S, \zeta(T))=+\infty$ for any $T \in \mathbb{R}$. Moreover, $\phi^{s,+}(0, \zeta(T))=\bar{\theta}$ and $\phi^{s,-}(0, \zeta(T))=\bar{\theta}-\pi$.

As for $\boldsymbol{W}^{\boldsymbol{u}}$, a similar situation occurs. Note first that assumption $\boldsymbol{G}_{\boldsymbol{u}}$ ensures that $W_{l_{u}}^{u}(T)$ exists for any $T \in \mathbb{R}$. Recall that $W_{l_{u}}^{u}(-\infty)$ coincides with the unstable manifold $M^{u}$ of the autonomous system (2.2) with $l=l_{u}$ and $g_{l_{u}}(x, t) \equiv g_{l_{u}}^{-\infty}(x)$, so $M^{u,+}$ and

\begin{tabular}{|l|l|l|l|l|l|}
\hline 220 & 2546 & $\mathbf{B}$ & $\begin{array}{l}\text { Dispatch: 28/12/2015 } \\
\text { Total pages: 27 } \\
\text { Disk Received } \\
\text { Disk Used } \square\end{array}$ & $\begin{array}{l}\text { Journal: Commun. Math. Phys. } \\
\text { Not Used } \square \\
\text { Corrupted } \square \\
\text { Mismatch } \square\end{array}$ \\
\hline
\end{tabular}


$M^{u,-}$ are unbounded spirals which rotate infinitely many times around the origin, see e.g. $[13,14,17]$ and Fig. 1.

Taking into account (3.2), Remark 2.1, Lemma 2.8 and the definition of polar coordinates (3.1) for a solution $\boldsymbol{x}_{l}:=\left(x_{l}, y_{l}\right)$ of (2.2), we easily conclude

Lemma 3.6. Let $\left(\boldsymbol{x}_{l_{u}}(t), z(t)\right)$ and $\left(\overline{\boldsymbol{x}}_{l_{u}}(t), z(t)\right)$ be trajectories in $\boldsymbol{W}^{\boldsymbol{u},+}$ and $\boldsymbol{W}^{\boldsymbol{u},-}$, respectively; let $\theta^{u,+}(t)$ and $\theta^{u,-}(t)$ be the angular coordinates of $\boldsymbol{x}_{l_{u}}(t)$ and $\overline{\boldsymbol{x}}_{l_{u}}(t)$. Then, $\lim _{t \rightarrow-\infty} \theta^{u,+}(t)=0$ and $\lim _{t \rightarrow-\infty} \theta^{u,-}(t)=-\pi$.

Reasoning as in the stable manifold case, we define two-variables parametrizations of $W^{u, \pm}$ as follows:

$$
\Sigma_{l_{u}}^{u, \pm}(U, z):[0,+\infty) \times[0,+\infty) \rightarrow \boldsymbol{W}^{u, \pm}
$$

such that $\Sigma_{l_{u}}^{u, \pm}(0, z)=(0,0, z)$ for any $z \geq 0$. Then, we introduce polar coordinates, by setting

$$
\Sigma_{l_{u}}^{u, \pm}(U, z)=\left(R_{l_{u}}^{u, \pm}(U, z) \cos \left(\phi^{u, \pm}(U, z)\right), R_{l_{u}}^{u, \pm}(U, z) \sin \left(\phi^{u, \pm}(U, z)\right), z\right) .
$$

Fix $T \in \mathbb{R}$, choose $Q^{ \pm} \in W_{l_{u}}^{u, \pm}(T)$, consider the trajectories $\boldsymbol{x}_{l_{u}}\left(t ; T, \boldsymbol{Q}^{ \pm}(T)\right)$ of (2.2): according to (3.1), we denote by $\theta^{u, \pm}(t)$ the angular coordinates of $\boldsymbol{x}_{l_{u}}\left(t ; T, Q^{ \pm}(T)\right)$.

With arguments analogous to the ones developed above in the study of the stable manifold we can reprove the analogous of Lemma 3.3; then, using also Lemmas 3.6 and 3.1 , we can state the following result.

Proposition 3.7. Take $T \in \mathbb{R}, Q^{ \pm}(T) \in W_{l_{u}}^{u, \pm}(T)$, let $U_{T}^{ \pm}>0$ be such that $\Sigma_{l_{u}}^{u, \pm}\left(U_{T}^{ \pm}, z(T)\right)=\left(Q^{ \pm}(T), z(T)\right)$, then the trajectories $\boldsymbol{x}_{l_{u}}\left(\cdot ; T, \boldsymbol{Q}^{+}(T)\right)$ and $\boldsymbol{x}_{l_{u}}\left(\cdot ; T, Q^{-}(T)\right)$ perform in the interval of time $(-\infty, T]$ the angles $\phi^{u,+}\left(U_{T}^{+}, z(T)\right)$ and $\phi^{u,-}\left(U_{T}^{-}, z(T)\right)+\pi$ around the origin, respectively.

Observe that $\Sigma_{l_{u}}^{u, \pm}(U, z(T))$ rotates clockwise on the coordinate axes as $U$ moves from 0 to $U_{T}^{ \pm}$, as well as the flows $\boldsymbol{x}_{l_{u}}\left(t ; T, Q^{ \pm}(T)\right)$ as $t$ moves from $-\infty$ to $T$. As a direct consequence, $\theta^{u,+}(T)=\phi^{u,+}\left(U_{T}^{+}, z(T)\right)<0$ and $\theta^{u,-}(T)=\phi^{u,-}\left(U_{T}^{-}, z(T)\right)<-\pi$.

As in the stable manifold case, we can prove the following lemma.

Lemma 3.8. $W_{l_{u}}^{u,+}(T)$ and $W_{l_{s}}^{s,-}(T)$ are spirals rotating clockwise starting from $(0,0)$, and they intersect the coordinate axes infinitely many times for any $T \in \mathbb{R}$.

As a direct consequence, we obtain that $\lim _{U \rightarrow+\infty} \phi^{u, \pm}(U, z(T))=-\infty$ for any $T \in \mathbb{R}$. Moreover, $\phi^{u,+}(0, z(T))=0$ and $\phi^{u,-}(0, z(T))=-\pi$.

Recalling the definition of $W_{l_{s}}^{u}(T)$ in (2.9), from a trivial topological argument we get the following result.

Lemma 3.9. $W_{l_{s}}^{u,+}(T)$ intersects $W_{l_{s}}^{s}(T)$ in a sequence of points $Q_{j}^{*,+}(T)$, for any $T \in \mathbb{R}$ and any $j \in \mathbb{N}$. Moreover, we can assume that $Q_{j}^{*,+}(T) \in W_{l_{s}}^{s,+}(T)$ if $j$ is even, while $Q_{j}^{*,+}(T) \in W_{l_{s}}^{s,-}(T)$ if $j$ is odd.

Proof. Fix $T \in \mathbb{R}$; taking into account the parametrization of $W_{l_{u}}^{u,+}(T)$ in (3.7), we obtain the following parametrization of $W_{l_{s}}^{u,+}(T)$

$$
\Sigma_{l_{s}}^{u,+}(U, z(T))=\left(R_{l_{s}}^{u,+}(U, z) \cos \left(\phi^{u,+}(U, z)\right), R_{l_{s}}^{u,+}(U, z) \sin \left(\phi^{u,+}(U, z)\right), z\right),
$$

\begin{tabular}{|l|l|l|l|l|l|}
\hline 220 & 254.6 & $\mathbf{B}$ & $\begin{array}{l}\text { Dispatch: 28/12/2015 } \\
\text { Total pages: 27 } \\
\text { Disk Received } \square \\
\text { Disk Used } \square\end{array}$ & $\begin{array}{l}\text { Journal: Commun. Math. Phys. } \\
\text { Not Used } \square \\
\text { Corrupted } \square \\
\text { Mismatch } \square\end{array}$ \\
\hline
\end{tabular}


where $R_{l_{s}}^{u, \pm}(U, z):=e^{-\left(\alpha_{l_{u}}-\alpha_{l_{s}}\right) T} R_{l_{u}}^{u, \pm}(U, z)$ and $z=z(T)$. Omitting, for simplicity, the dependence on $T$, according to (3.3) and (3.7), we define the curves $\Gamma^{s, \pm}(S)$ : $[0,+\infty) \rightarrow \mathbb{R} \times[0,+\infty)$ and $\Gamma^{u, \pm}(U):[0,+\infty) \rightarrow \mathbb{R} \times[0,+\infty)$ by setting

$$
\Gamma^{s, \pm}(S):=\left(\phi^{s, \pm}(S), R_{l_{s}}^{s, \pm}(S)\right), \text { and } \quad \Gamma^{u, \pm}(U):=\left(\phi^{u, \pm}(U), R_{l_{s}}^{u, \pm}(U)\right) .
$$

Note that the curves $\Gamma^{s, \pm}$ and $\Gamma^{u, \pm}$ are the liftings of $W_{l_{s}}^{s, \pm}(T)$ and $W_{l_{s}}^{u, \pm}(T)$, respectively. We recall that $\Gamma^{u,+}(0)=(0,0)$ and $\lim _{U \rightarrow+\infty} \phi^{u,+}(U)=-\infty$. In particular, the image of $\Gamma^{u,+}$ splits the stripe $\{(\theta, \rho) \mid \theta \in \mathbb{R}, \rho \geq 0\}$ into two open sets, say $A^{l}$ and $A^{r}$. We denote by $A^{r}$ the set on the right of $A^{l}$ in the coordinate system with horizontal $\theta$-axis. Let us define the curves:

$$
\Gamma_{2 k}^{s}(S):=\left(\phi^{s,+}(S)-2 \pi k, R_{l_{s}}^{s,+}(S)\right), \quad \Gamma_{2 k+1}^{s}(S):=\left(\phi^{s,-}(S)-2 \pi k, R_{l_{s}}^{s,-}(S)\right),
$$

for $k \in \mathbb{N}$, so that $\Gamma_{0}^{s}(S)=\Gamma^{s,+}(S), \Gamma_{1}^{s}(S)=\Gamma^{s,-}(S)$ and $\Gamma_{j}^{s}$ is a translation of $\Gamma^{s,+}$ for $j$ even and of $\Gamma^{s,-}$ for $j$ odd. Note that the curve $\Gamma_{j}^{s}$ cannot intersect $\Gamma_{k}^{s}$ if $j \neq k$, since $W_{l_{s}}^{s}(T)$ cannot have self-intersections. According to this notation, $\Gamma_{j}^{s}(0)=$ $(\bar{\theta}-j \pi, 0) \in A^{l}$, and $\lim _{S \rightarrow+\infty}\left[\phi^{s, \pm}(S)-\pi j\right]=+\infty$, for any $j \geq 0$. Thus, from a continuity argument, it follows that for any $j \geq 0$, there is at least one $S>0$ such that $\Gamma_{j}^{S}(S)$ lies on the graph of $\Gamma^{u,+}$, i.e. the graphs of $\Gamma_{j}^{S}(\cdot)$ and $\Gamma^{u,+}(\cdot)$ intersect at least in a point. Let us set

$$
U_{j}^{*}:=\min \left\{U>0 \mid \Gamma^{u,+}(U) \in \Gamma_{j}^{s}(] 0, \infty[)\right\},
$$

and let $S_{j}^{*}>0$ be the value such that $\Gamma_{j}^{s}\left(S_{j}^{*}\right)=\Gamma^{u,+}\left(U_{j}^{*}\right)$. Let us now define

$$
\begin{aligned}
& \Omega_{j}^{*,+}:=\Gamma^{u,+}\left(U_{j}^{*}\right)=\left(\phi^{u,+}\left(U_{j}^{*}\right), R_{l_{s}}^{u,+}\left(U_{j}^{*}\right)\right), \\
& Q_{j}^{*,+}:=\left(R_{l_{s}}^{u,+}\left(U_{j}^{*}\right) \cos \left[\phi^{u,+}\left(U_{j}^{*}\right)\right], R_{l_{s}}^{u,+}\left(U_{j}^{*}\right) \sin \left[\phi^{u,+}\left(U_{j}^{*}\right)\right]\right) .
\end{aligned}
$$

By construction, $Q_{j}^{*,+} \in W_{l_{s}}^{u,+}(T) \cap W_{l_{s}}^{s}(T)$. Moreover, $Q_{j}^{*,+} \neq Q_{k}^{*,+}$ for $k \neq j$, since $W_{l_{s}}^{u}(T)$ cannot have self-intersections.

Remark 3.10. By construction the sequence $U_{k}^{*}$ is increasing in $k \in \mathbb{N}$, since $\boldsymbol{W}^{\boldsymbol{u}}$ cannot have self-intersections.

In fact, the sequences $S_{2 k}^{*}$ and $S_{2 k+1}^{*}$ are increasing too. Since this property will not be used in the paper, its proof is left to the interested reader.

Lemma 3.11. Let $u\left(r, d_{j}^{*}\right)$ be the solution of (1.2) corresponding to $\boldsymbol{x}_{l_{s}}\left(t ; T, Q_{j}^{*,+}\right)$. Then, $u\left(r, d_{j}^{*}\right)$ is a regular, fast decay solution with exactly $j$ non-degenerate zeroes. In particular, $u\left(r, d_{0}^{*}\right)$ is a positive solution.

The sequence $d_{j}^{*}$ is increasing and $d_{j}^{*} \nearrow+\infty$.

Proof. By construction, $x_{l_{s}}\left(t ; T, Q_{j}^{*,+}(T)\right)$ is a homoclinic trajectory of (2.2), and the corresponding solution $u\left(r, d_{j}^{*}\right)$ of (1.2) is regular and has fast decay. Note that $\phi^{s,+}\left(S_{j}^{*}\right)-$ $j \pi=\phi^{u,+}\left(U_{j}^{*}\right)$ if $j$ is even, and $\phi^{s,-}\left(S_{j}^{*}\right)-(j-1) \pi=\phi^{u,+}\left(U_{j}^{*}\right)$ if $j$ is odd. Thus, $\boldsymbol{x}_{l_{s}}\left(\cdot ; T, Q_{j}^{*,+}(T)\right)$ performs in $[T,+\infty)$ the angle $\left(\bar{\theta}-\phi^{u,+}\left(U_{j}^{*}\right)-j \pi\right)$ around the origin

\begin{tabular}{|l|l|l|l|l|l|}
\hline $\mathbf{2} 2 \mathbf{2 0}$ & $\mathbf{2 5 4 6}$ & $\mathbf{B}$ & $\begin{array}{l}\text { Dispatch: 28/12/2015 } \\
\text { Total pages: 27 } \\
\text { Disk Received } \\
\text { Disk Used } \square\end{array}$ & $\begin{array}{l}\text { Journal: Commun. Math. Phys. } \\
\text { Not Used } \square \\
\text { Corrupted } \square \\
\text { Mismatch } \square\end{array}$ \\
\hline Jour. No & Ms. No.
\end{tabular}


by Proposition 3.4, while it performs in $(-\infty, T]$ the angle $\phi^{u,+}\left(U_{j}^{*}\right)$ by Proposition 3.7. Therefore, $\boldsymbol{x}_{l_{s}}\left(t ; T, \boldsymbol{Q}_{j}^{*,+}(T)\right)$ performs for $t \in \mathbb{R}$ the angle

$$
\bar{\theta}-\phi^{u,+}\left(U_{j}^{*}\right)-j \pi+\phi^{u,+}\left(U_{j}^{*}\right)=\bar{\theta}-j \pi,
$$

which, in particular, is $T$-independent. This implies that $\boldsymbol{x}_{l_{s}}\left(t ; T, Q_{j}^{*,+}(T)\right)$ for $t \in \mathbb{R}$ makes exactly $j$ semi-rotations clockwise around the origin (minus $\bar{\theta} \in(-\pi / 2,0)$ ), so $u\left(r, d_{j}^{*}\right)$ has exactly $j$ non-degenerate zeroes for $r \geq 0$.

The monotonicity of $d_{j}^{*}$ follows from the monotonicity of $U_{j}^{*}$ established in Remark 3.10 and from Lemma 2.10.

Let us now prove that $U_{j}^{*}$ is unbounded. Assume, by contradiction, that $U_{j}^{*} \nearrow \bar{U}<$ $\infty$ as $j \rightarrow+\infty$. If we set $\overline{\boldsymbol{Q}}=\Sigma_{l_{s}}^{u,+}(\bar{U}, z(T))$, we also have $\overline{\boldsymbol{Q}} \in W_{l_{s}}^{s,+}(T)$ and $\overline{\mathbf{Q}} \in W_{l_{s}}^{s,-}(T)$, a contradiction. Hence, $U_{j}^{*}$ is unbounded, and, by Lemma $2.10, d_{j}^{*}$ is unbounded too.

Remark 3.12. We emphasize that, a priori, the curves $\Gamma^{u,+}$ and $\Gamma_{j}^{s}$ may have several intersections: in this case we have many regular solutions with fast decay and exactly $j$ zeroes.

Analogous versions of Lemmas 3.9 and 3.11 can be written for $W_{l_{s}}^{u,-}(T)$. As for $W_{l_{s}}^{u,+}$, we set

$$
\begin{aligned}
\tilde{U}_{j}^{*} & :=\min \left\{U>0 \mid \Gamma^{u,-}(U) \in \Gamma_{j+1}^{s}(] 0,+\infty[)\right\}, \\
\Omega_{j}^{*,-} & :=\Gamma^{u,-}\left(\tilde{U}_{j}^{*}\right)=\left(\phi^{u,-}\left(\tilde{U}_{j}^{*}\right), R_{l_{s}}^{u,-}\left(\tilde{U}_{j}^{*}\right)\right), \\
Q_{j}^{*,-} & :=\left(R_{l_{s}}^{u,-}\left(\tilde{U}_{j}^{*}\right) \cos \left[\phi^{u,-}\left(\tilde{U}_{j}^{*}\right)\right], R_{l_{s}}^{u,-}\left(\tilde{U}_{j}^{*}\right) \sin \left[\phi^{u,-}\left(\tilde{U}_{j}^{*}\right)\right]\right) .
\end{aligned}
$$

Similarly to (3.7), we define the curves

$$
\begin{aligned}
\Gamma_{2 k}^{u}(U) & :=\left(\phi^{u,+}(U)-2 \pi k, R_{l_{u}}^{u,+}(U)\right), \\
\Gamma_{2 k+1}^{u}(U) & :=\left(\phi^{u,-}(U)-2 \pi k, R_{l_{u}}^{u,-}(U)\right),
\end{aligned}
$$

for $k \in \mathbb{N}$, which, combined with (3.9), determine a net on the $(\theta, \rho)$-plane. Here and below, we omit the dependence on $T$ of all the variables in (3.10), when no confusion arises.

For any $t \in \mathbb{R}$ and any $j \in \mathbb{N}$, denote by $\bar{\Gamma}^{u,+}(t), \bar{\Gamma}^{u,-}(t), \bar{\Gamma}_{j}^{u}(t), \bar{\Gamma}_{j}^{s}(t)$ the graphs of $\Gamma^{u,+}(\cdot, t), \Gamma^{u,-}(\cdot, t), \Gamma_{j}^{u}(\cdot, t), \Gamma_{j}^{s}(\cdot, t)$, respectively.

Moreover, set $\bar{\Gamma}^{u}(t):=\cup_{j \in \mathbb{N}} \bar{\Gamma}_{j}^{u}(t), \bar{\Gamma}^{s}(t):=\bigcup_{j \in \mathbb{N}} \bar{\Gamma}_{j}^{s}(t)$ and $\bar{\Gamma}(t):=\bar{\Gamma}^{u}(t) \cup \bar{\Gamma}^{s}(t)$.

We emphasize that, by construction, a key invariance property holds. More precisely, let $\bar{\Omega}_{\bar{Q}}=(\bar{\theta}, \bar{\rho}) \in \mathbb{R} \times(0,+\infty)$ be the polar coordinates of $\overline{\boldsymbol{Q}}$ and denote by $\Omega\left(t ; T, \bar{\Omega}_{\bar{Q}}\right)=\left(\theta(t ; T, \bar{\Omega} \overline{\boldsymbol{Q}}), \rho\left(t ; T, \bar{\Omega}_{\overline{\boldsymbol{Q}}}\right)\right)$ the polar coordinates of $\boldsymbol{x}_{l_{s}}(t ; T, \overline{\boldsymbol{Q}})$ (assuming that $\Omega\left(t ; T, \bar{\Omega}_{\bar{Q}}\right)$ is continuous and $\left.\Omega\left(T ; T, \bar{\Omega}_{\bar{Q}}\right)=\bar{\Omega}_{\bar{Q}}\right)$.

Lemma 3.13. If $\bar{\Omega} \in \bar{\Gamma}^{u,+}(T), \hat{\Omega} \in \bar{\Gamma}^{u,-}(T)$ and $\tilde{\Omega} \in \bar{\Gamma}_{j}^{s}(T)$, then $\Omega(t ; T, \bar{\Omega}) \in$ $\bar{\Gamma}^{u,+}(t), \Omega(t ; T, \hat{\Omega}) \in \bar{\Gamma}^{u,-}(t)$ and $\Omega(t ; T, \tilde{\Omega}) \in \bar{\Gamma}_{j}^{s}(t)$, for any $t \in \mathbb{R}$. Moreover, $\lim _{t \rightarrow-\infty} \theta(t ; T, \bar{\Omega})=0, \lim _{t \rightarrow-\infty} \theta(t ; T, \bar{\Omega})=-\pi$ and $\lim _{t \rightarrow+\infty} \theta(t ; T, \bar{\Omega})=(\bar{\theta}-$ $j \pi, 0$ ). 
Proof. Let $\bar{\Omega}$ be the polar coordinates of $\overline{\boldsymbol{Q}}$. Moreover, let $\boldsymbol{x}_{l_{s}}(t, T, \overline{\boldsymbol{Q}})$ and $\boldsymbol{x}_{l_{u}}(t, T, \overline{\boldsymbol{S}})$ be the trajectories of (2.2) with $\bar{S}:=\bar{Q} e^{\left(\alpha_{l_{u}}-\alpha_{l_{s}}\right) T}$ and let $u(r)$ be the solution of (1.2) corresponding to $\Omega(t ; T, \bar{\Omega})$. Then, by construction, $\boldsymbol{x}_{l_{s}}(t ; T, \overline{\boldsymbol{Q}}) \in W_{l_{s}}^{u}(t), \boldsymbol{x}_{l_{u}}(t ; T, \overline{\boldsymbol{S}}) \in$ $W_{l_{u}}^{u}(t)$ and $u(r)$ is a regular solution. Hence, $\Omega(t ; T, \bar{\Omega}) \in \bar{\Gamma}^{u,+}(t)$ for any $t \in \mathbb{R}$. Furthermore, $\lim _{t \rightarrow-\infty} \boldsymbol{x}_{l_{u}}(t ; T, \overline{\boldsymbol{S}})=(0,0)$, and $\boldsymbol{x}_{l_{u}}$ approaches the origin tangent to the $x$ positive semi-axis, so $\lim _{t \rightarrow-\infty} \theta(t ; T, \bar{\Omega})=0$.

The proofs concerning $\hat{\Omega}$ and $\tilde{\Omega}$ are analogous and follow by Lemmas 3.6 and 3.2, respectively.

We now introduce some sets which will play a fundamental role in the proof of our main theorem. In particular, we will devote our attention on the stripe between $\bar{\Gamma}^{u,+}$ and $\bar{\Gamma}^{u,-}$.

Denote by $\boldsymbol{A}^{u}(t)$ the open stripe in the $(\theta, \rho)$-plane between $\bar{\Gamma}^{u,+}(t)$ and $\bar{\Gamma}^{u,-}(t)$; denote by $\boldsymbol{B}_{j}^{s}(t)$ the open stripe between $\bar{\Gamma}_{j-1}^{s}(t)$ and $\bar{\Gamma}_{j}^{s}(t)$. Finally, define $\boldsymbol{K}_{\boldsymbol{j}}(t):=$ $\boldsymbol{A}^{\boldsymbol{u}}(t) \cap \boldsymbol{B}_{j}^{s}(t)$. From the first part of Lemma 3.13, it is easy to deduce that these sets satisfy the invariant property.

Lemma 3.14. If $\bar{\Omega} \in A^{u}(T), \boldsymbol{B}_{j}^{s}(T), \boldsymbol{K}_{j}(T)$, respectively, then $\Omega(t ; T, \bar{\Omega}) \in A^{u}(t)$, $\boldsymbol{B}_{\boldsymbol{j}}^{\boldsymbol{s}}(t), \boldsymbol{K}_{\boldsymbol{j}}(t)$ for any $t \in \mathbb{R}$, respectively.

Remark 3.15. If $\bar{\Omega} \in \boldsymbol{K}_{j}(T)$, then $\theta(t ; T, \bar{\Omega}) \in\left(-j \pi-\frac{\pi}{2}, 0\right)$ for any $t \in \mathbb{R}$. Indeed, by Lemma 3.1 combined with Propositions 3.4 and 3.7 we easily deduce that, for any $t \in \mathbb{R}, \bar{\Gamma}^{u,+}(t)$ cannot intersect the $\theta=0$ axis, while $\bar{\Gamma}_{j}^{s}(t)$ cannot intersect the vertical line $\theta=-\left(j \pi+\frac{\pi}{2}\right)$. Taking into account that $\boldsymbol{K}_{\boldsymbol{j}}(t)$ is contained in the region bounded by $\bar{\Gamma}^{u,+}(t)$ on the right, $\bar{\Gamma}_{j}^{s}(t)$ on the left and by the $\rho=0$ axis from below, the thesis follows.

Denote by $\Lambda^{+}(-\infty)=\left(\phi^{+}(-\infty), R^{+}(-\infty)\right)$ and $\Lambda^{ \pm}(+\infty)=\left(\phi^{ \pm}(+\infty), R^{ \pm}(+\infty)\right)$ the polar coordinates of the critical points $\boldsymbol{P}^{+}(-\infty) \in W_{l_{u}}^{u}(-\infty)$ and $\boldsymbol{P}^{ \pm}(+\infty) \in$ $W_{l_{s}}^{s}(+\infty)$, respectively. According to the adopted notation and recalling that $\bar{\theta}=-\arctan (n-2) \in(-\pi / 2,0)$, we know that $\phi^{+}( \pm \infty) \in(\bar{\theta}, 0)$ and $\phi^{-}(+\infty) \in$ $(\bar{\theta}-\pi,-\pi)$.

Finally define $\Lambda_{2 k}^{+}(+\infty):=\left(\phi^{+}(+\infty)-2 k \pi, R^{+}(+\infty)\right)$ and $\Lambda_{2 k+1}^{-}(+\infty):=$ $\left(\phi^{-}(+\infty)-2 k \pi, R^{-}(+\infty)\right)$.

In order to give a first version of the proof of Theorem 2.4, we introduce two simplifying assumptions, which allow us to explain the main ideas avoiding technicalities. Such assumptions will be removed later on.

$\mathbf{H}^{ \pm}$For any $j \in \mathbb{N}$ there is a unique intersection between $\bar{\Gamma}^{u, \pm}(T)$ and $\bar{\Gamma}_{j}^{s}(T)$.

Remark 3.16. Consider $f$ of type (1.3) and assume $r k^{\prime}(r) / k(r)$ decreasing. Then, $\mathbf{H}^{+}$ and $\mathbf{H}^{-}$are satisfied.

Proof. Yanagida in [39, Theorem 1] proved the existence of the sequence $d_{j}^{*}$ of Lemma 3.11 under the assumptions of Remark 3.16, and showed that if $u(r)$ and $v(r)$ are distinct G.S. with f.d., then they have a different number of zeroes. On the contrary, from the proof of Lemma 3.11, it follows that two intersections between $\bar{\Gamma}^{u,+}(T)$ and $\bar{\Gamma}_{j}^{s}(T)$ correspond to two G.S. with f.d. with exactly $j$ zeroes. So, this intersection is unique and $\mathbf{H}^{+}$follows. To complete the proof, we observe that an analogous argument works for $\bar{\Gamma}^{u,-}(T)$.

\begin{tabular}{|l|l|l|l|l|l|}
\hline $\mathbf{2} 2 \mathbf{2 0}$ & $\mathbf{2 5 4 6}$ & $\mathbf{B}$ & $\begin{array}{l}\text { Dispatch: 28/12/2015 } \\
\text { Total pages: 27 } \\
\text { Disk Received } \\
\text { Disk Used } \square\end{array}$ & $\begin{array}{l}\text { Journal: Commun. Math. Phys. } \\
\text { Not Used } \square \\
\text { Corrupted } \square \\
\text { Mismatch } \square\end{array}$ \\
\hline Jour. No & Ms. No.
\end{tabular}




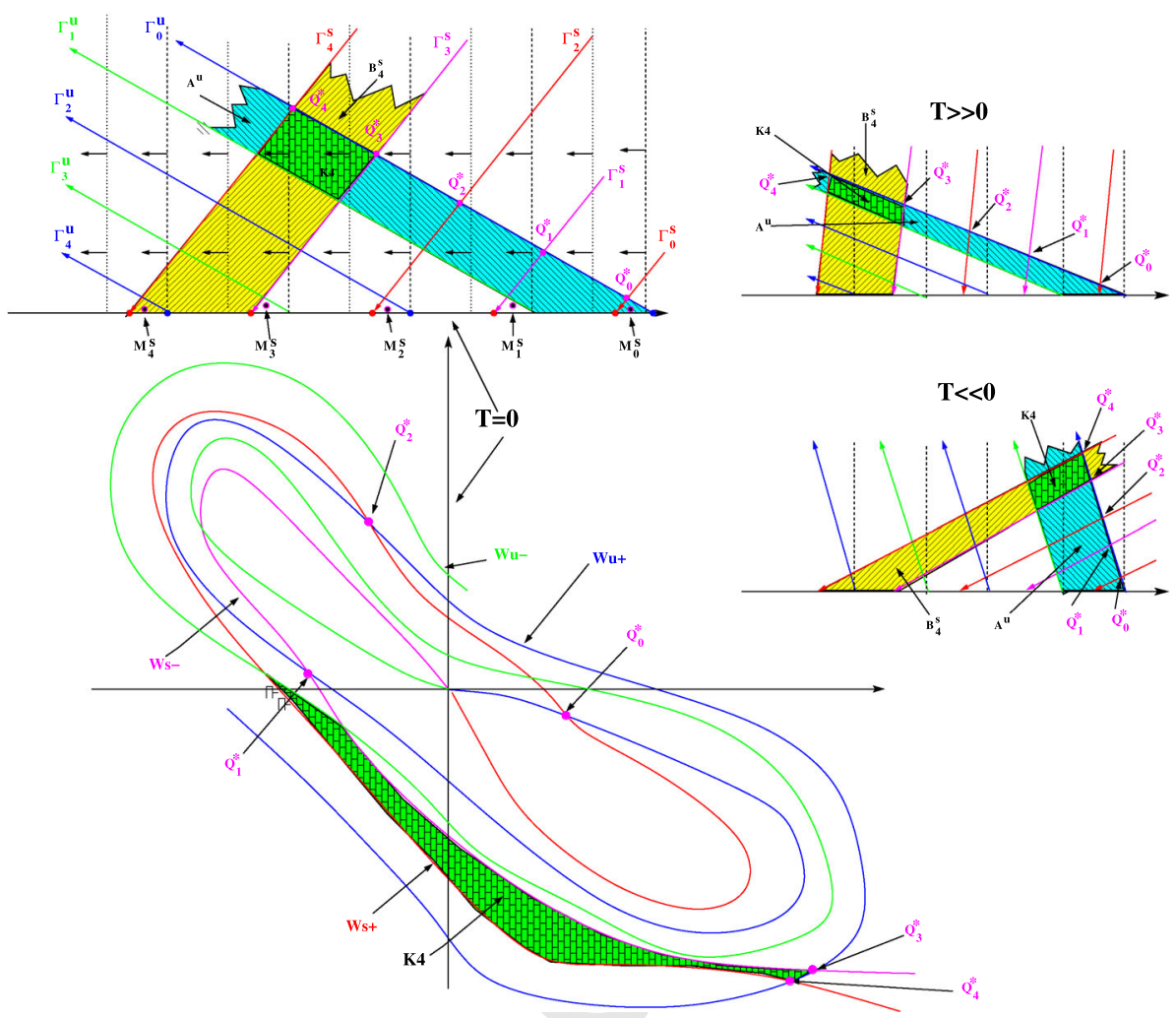

Fig. 2. Sketch of the proofs of Lemma 3.11, Propositions 3.19 and 3.20, assuming $\mathbf{H}^{ \pm}$

The proof of existence of G.S. with s.d, S.G.S. with f.d., and S.G.S. with s.d. is obtained with a geometrical argument developed on Figs. 2 and 3. More precisely, Fig. 2 refers to the case where $\mathbf{H}^{ \pm}$hold, while Fig. 3 refers to the general case.

We now show that if $\bar{\Omega} \in K_{j}(T)$, then the corresponding solution $u(r)$ of (1.2) is singular-slow decay and has exactly $j$ zeroes, under assumptions $\mathbf{H}^{ \pm}$. To this purpose, we need some preliminary lemmas.

Lemma 3.17. Assume $\mathbf{H}^{+}$and $\mathbf{H}^{-}$. Consider $\hat{\Omega} \in \boldsymbol{B}_{2 k}^{s}(T), \tilde{\Omega} \in \boldsymbol{B}_{2 k+1}^{s}(T)$, then $\lim _{t \rightarrow \infty} \Omega(t ; T, \hat{\Omega})=\Lambda_{2 k}^{+}(+\infty), \lim _{t \rightarrow \infty} \Omega(t ; T, \tilde{\Omega})=\Lambda_{2 k+1}^{-}(+\infty)$ and the corresponding solutions $\hat{u}(r), \tilde{u}(r)$ of (1.2) have slow decay and are definitely positive and definitely negative for $r$ large, respectively.

Proof. Consider $\Omega(t ; T, \bar{\Omega})$ with $\bar{\Omega} \in \boldsymbol{B}_{j}^{\boldsymbol{s}}(T)$, and let $\boldsymbol{x}_{l_{s}}(t ; T, \overline{\boldsymbol{Q}})$ be the corresponding trajectory of (2.2), and $\bar{u}(r)$ the corresponding solution of (1.2). According to the invariance property stated in Lemma 3.13, $\boldsymbol{B}_{j}^{s}(t) \cap \bar{\Gamma}^{s}(t)=\emptyset$ for every $t \in \mathbb{R}$, so $\bar{u}(r)$ cannot be a fast decay solution. Moreover, according to Lemma 3.14, $\Omega(t ; T, \bar{\Omega}) \in \boldsymbol{B}_{j}^{\boldsymbol{s}}(t)$ for every $t \in \mathbb{R}$, so $\bar{u}(r)$ cannot rotate indefinitely as $r \rightarrow+\infty$. Hence, from Lemma 2.9 we see that $\bar{u}(r)$ has slow decay.

Focusing now on $W_{l_{s}}^{s}(\tau)$, note that the two counterclockwise spirals $W_{l_{s}}^{s,+}(\tau)$ and $W_{l_{s}}^{s,-}(\tau)$ do not intersect each other and divide the $(x, y)$-plane into two connected

\begin{tabular}{|l|l|l|l|l|l|}
\hline 220 & 2546 & $\mathbf{B}$ & $\begin{array}{l}\text { Dispatch: 28/12/2015 } \\
\text { Total pages: 27 } \\
\text { Disk Received } \square \\
\text { Disk Used } \square\end{array}$ & $\begin{array}{l}\text { Journal: Commun. Math. Phys. } \\
\text { Not Used } \square \\
\text { Corrupted } \square \\
\text { Mismatch } \square\end{array}$ \\
\hline
\end{tabular}




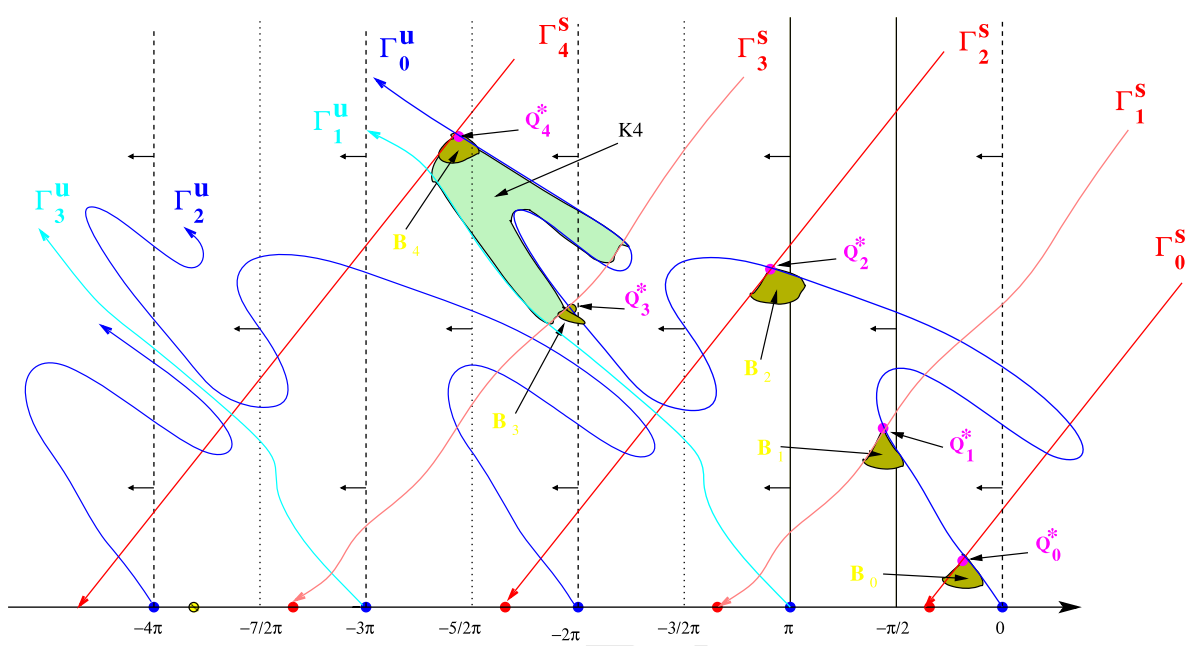

Fig. 3. Sketch of the proofs of Lemma 3.11, Propositions 3.22 and 3.23, when $\mathbf{H}^{ \pm}$are removed

open sets, say $\boldsymbol{M}_{0}^{s}(t)$ and $\boldsymbol{M}_{1}^{s}(t)$, each of them containing only one critical point at $t \rightarrow+\infty$, say $\boldsymbol{P}^{+}(+\infty) \in \boldsymbol{M}_{0}^{s}(+\infty)$ and $\boldsymbol{P}^{-}(+\infty) \in \boldsymbol{M}_{1}^{s}(+\infty)$. Note that, by definition and according to Lemma $3.13, \boldsymbol{B}_{2 k}^{s}(+\infty)$ and $\boldsymbol{B}_{2 k+1}^{s}(+\infty)$ represent a parametrization in polar coordinates of $\boldsymbol{M}_{0}^{s}(+\infty)$ and $\boldsymbol{M}_{1}^{s}(+\infty)$, respectively. From Lemma 2.9, we conclude that $\Omega(t ; T, \bar{\Omega})$ converges to the only critical point in $\boldsymbol{B}_{j}^{\boldsymbol{s}}(+\infty)$. More precisely, $\lim _{t \rightarrow \infty} \Omega(t ; T, \hat{\Omega})=\Lambda_{2 k}^{+}(+\infty), \lim _{t \rightarrow \infty} \Omega(t ; T, \tilde{\Omega})=\Lambda_{2 k+1}^{-}(+\infty)$, and the thesis follows.

Recalling that Kelvin inversion allows us to translate results for slow decay solutions into results for singular solutions, from Lemma 3.17 combined with Lemma 3.13, we easily deduce the following result.

Lemma 3.18. Assume $\mathbf{H}^{+}$and $\mathbf{H}^{-}$. If $\bar{\Omega} \in A^{\boldsymbol{u}}(T)$, then $\lim _{t \rightarrow-\infty} \theta(t ; T, \bar{\Omega})=\phi^{+}(-\infty)$. The solution $\bar{u}(r)$ of $(1.2)$ corresponding to $\Omega(t ; T, \bar{\Omega})$ is singular and is definitely positive for $r$ small.

The required multiplicity result for initial data in $\boldsymbol{K}_{\boldsymbol{j}}(T)$ follows.

Proposition 3.19. Assume $\mathbf{H}^{+}$and $\mathbf{H}^{-}$. If $\bar{\Omega} \in \boldsymbol{K}_{\boldsymbol{j}}(T)$, then the solution $\bar{u}(r)$ of (1.2) corresponding to $\Omega(t ; T, \bar{\Omega})$ is singular-slow decay and has exactly $j$ zeroes.

Proof. By combining Lemmas 3.17 and 3.18 with the definition of $\boldsymbol{K}_{\boldsymbol{j}}(t)$, we deduce that $\bar{u}(r)$ is a singular-slow decay solution.

If $\bar{\Omega} \in K_{2 k}(T)$, then $\lim _{t \rightarrow \infty} \theta(t ; T, \hat{\Omega})=\phi^{+}(+\infty)-2 k \pi \in(\bar{\theta}-2 k \pi,-2 k \pi)$ and $\lim _{t \rightarrow-\infty} \theta(t ; T, \hat{\Omega})=\phi^{+}(-\infty) \in(\bar{\theta}, 0)$. Hence, $\Omega(t ; T, \bar{\Omega})$ intersects the vertical line $\theta=i \pi-\frac{\pi}{2}$ for any $i \in\{1, \ldots, 2 k\}$. Each of these $2 k$ intersections corresponds to a zero of $\boldsymbol{x}_{l_{s}}(\cdot ; T, \overline{\boldsymbol{Q}})$, where $\boldsymbol{x}_{l_{s}}(\cdot ; T, \overline{\boldsymbol{Q}})$ is the trajectory of $(2.2)$ and $\bar{\Omega}$ are the polar coordinates of $\overline{\boldsymbol{Q}}$. The exactness of the number of zeroes is a direct consequence of Lemma 3.1.

With the same argument we see that if $\bar{\Omega} \in K_{2 k+1}(T)$, then $\bar{u}(r)$ has exactly $2 k+1$ zeroes, so the goal is achieved.

ニ


We now concentrate on regular-slow decay solutions. To this aim, we set

$$
\boldsymbol{a}_{j}^{\boldsymbol{u}}(T):=\left\{\Gamma^{u,+}(U, T) \mid U_{j-1}^{*}(T) \leq U \leq U_{j}^{*}(T)\right\},
$$

so that $\boldsymbol{a}_{j}^{\boldsymbol{u}}(T)$ is the arc of $\bar{\Gamma}^{u,+}(T)$ between $\Omega_{j-1}^{*,+}(T)$ and $\Omega_{j}^{*,+}(T)$.

Given a path $A$, let us denote by $\AA$ the path $A$ without endpoints. Notice that, by definition, $\stackrel{\circ}{a}_{j}^{u} \subseteq \boldsymbol{B}_{j}^{s} \cap \bar{\Gamma}^{u,+}$. Hence, the following result holds.

Proposition 3.20. Assume $\mathbf{H}^{+}$and $\mathbf{H}^{-}$. If $\bar{\Omega} \in \stackrel{\circ}{\boldsymbol{a}}_{j}^{\boldsymbol{u}}(T)$, then for every $d \in\left(d_{j-1}^{*}, d_{j}^{*}\right)$ the solution $\bar{u}(r, d)$ of $(1.2)$ corresponding to $\Omega(t ; T, \bar{\Omega})$ is regular-slow decay and has exactly $j$ zeroes.

Proof. The proof follows by combining Lemma 3.13 with Lemma 3.17. As far as the number of zeros of $\bar{u}(r, d)$ is concerned, we just need to observe that $\lim _{t \rightarrow \infty} \theta(t ; T, \bar{\Omega}) \in$ $(\bar{\theta}-j \pi,-j \pi)$ and $\lim _{t \rightarrow-\infty} \theta(t ; T, \bar{\Omega})=0$, whenever $\bar{\Omega} \in \stackrel{\circ}{\boldsymbol{a}} \boldsymbol{u}_{j}(T)$. The thesis easily follows.

Note that Theorem 2.4 is an immediate consequence of Propositions 3.19 and 3.20 combined with Lemma 3.11. Recalling that Kelvin inversion enables us to convert results for regular solutions into results for fast decay solutions, we easily deduce that all the solutions of (1.2) have a structure of type Mix with $d_{j}^{*}=d_{j+1}$ for any $j \geq 0$.

Remark 3.21. We emphasize that assumption $\mathbf{H}^{+}$implies that $d_{j}^{*}=d_{j+1}$.

Note that this equality has been proven by Yanagida [39] in Theorem A under the monotonicity assumption on $\frac{r k^{\prime}(r)}{k(r)}$.

Now we remove assumptions $\mathbf{H}^{ \pm}$to provide an exhaustive proof of Theorem 2.4. We need to adapt Propositions 3.19 and 3.20 to this more general setting.

We recall that $\Omega_{j}^{*,+}(T):=\left(\theta_{j}^{*}(T), \rho_{j}^{*}(T)\right)$ are the polar coordinates of $Q_{j}^{*,+}(T)$. For every $\delta>0$, we define

$$
B_{j}(T, \delta):=\left\{\Omega=(\theta, \rho) \in \boldsymbol{K}_{j}(T):\left|\Omega-\Omega_{j}^{*,+}(T)\right|<\delta\right\},
$$

where $|\Omega|=\sqrt{\theta^{2}+\rho^{2}}$. Note that in the absence of assumptions $\mathbf{H}^{ \pm}$, the set $\boldsymbol{K}_{\boldsymbol{j}}(T)$ can be disconnected. Hence, we choose $\delta>0$ small enough to ensure that $B_{j}(T, \delta)$ is a connected set in $\boldsymbol{K}_{j}(T)$ and there exist $U_{j}(\delta) \in\left(U_{j-1}^{*}(T), U_{j}^{*}(T)\right), S_{j}(\delta) \in$ $\left(S_{j-1}^{*}(T), S_{j}^{*}(T)\right)$ such that the border $\partial B_{j}(T, \delta)$ of $B_{j}(T, \delta)$ is made up by $\Gamma^{u,+}\left(\left[U_{j}(\delta), U_{j}^{*}(T)\right], T\right), \Gamma_{j}^{s}\left(\left[S_{j}(\delta), S_{j}^{*}(T)\right], T\right)$ and a curve connecting them. More precisely,

$$
\partial B_{j}(T, \delta) \cap \bar{\Gamma}(T)=\Gamma^{u,+}\left(\left[U_{j}(\delta), U_{j}^{*}(T)\right], T\right) \cup \Gamma_{j}^{s}\left(\left[S_{j}(\delta), S_{j}^{*}(T)\right], T\right),
$$

where $\Gamma^{u,+}\left(\left[U_{j}(\delta), U_{j}^{*}(T)[, T) \cap \bar{\Gamma}^{s}(T)=\emptyset, \Gamma_{j}^{s}\left(\left[S_{j}(\delta), S_{j}^{*}(T)[, T) \cap \bar{\Gamma}^{u}(T)=\emptyset\right.\right.\right.\right.$.

Let us denote by $\operatorname{cl}(B)$ the closure of the set $B$.

We are now in position to state a revised version of Proposition 3.19, independent of conditions $\mathbf{H}^{ \pm}$.

Proposition 3.22. There exists $\bar{\delta}>0$ such that for every $\bar{\Omega} \in B_{j}(T, \bar{\delta})$, then the solution $\bar{u}(r)$ of (1.2) corresponding to $\Omega(t ; T, \bar{\Omega})$ is singular-slow decay and has exactly $j$ zeroes.

\begin{tabular}{|l|l|l|l|l|l|}
\hline 220 & $\mathbf{2 5 4 6}$ & $\mathbf{B}$ & $\begin{array}{l}\text { Dispatch: 28/12/2015 } \\
\text { Total pages: 27 } \\
\text { Disk Received } \\
\text { Disk Used } \square\end{array}$ & $\begin{array}{l}\text { Journal: Commun. Math. Phys. } \\
\text { Not Used } \square \\
\text { Corrupted } \\
\text { Mismatch }\end{array}$ \\
\hline Jour. No
\end{tabular}


Proof. By Lemma 3.13, $\lim _{t \rightarrow-\infty} \theta_{j}^{*}(t)=0$ and $\lim _{t \rightarrow+\infty} \theta_{j}^{*}(t)=\bar{\theta}-j \pi$.

Recalling that $\bar{\theta} \in(-\pi / 2,0)$, we deduce the existence of $\mathcal{T}_{j}>0$ such that $\theta_{j}^{*}\left(-\mathcal{T}_{j}\right) \in(-\pi / 2,0)$ and $\theta_{j}^{*}\left(-\mathcal{T}_{j}\right) \in\left(-j \pi-\frac{\pi}{2},-j \pi\right)$. Hence, using a continuity argument and taking into account Remark 3.15, we can choose $\varepsilon>0$ small enough to guarantee that there is $\bar{\delta}=\bar{\delta}(j, \varepsilon)>0$ such that

$$
\begin{aligned}
\left|\Omega(t ; T, \bar{\Omega})-\Omega_{j}^{*,+}(t)\right|<\varepsilon & \forall \bar{\Omega} \in \operatorname{cl}\left(B_{j}(T, \bar{\delta})\right),|t|<\mathcal{T}_{j}, \\
-\frac{\pi}{2}<\theta\left(-\mathcal{T}_{j} ; T, \bar{\Omega}\right)<0, & -j \pi-\frac{\pi}{2}<\theta\left(\mathcal{T}_{j} ; T, \bar{\Omega}\right)<-j \pi .
\end{aligned}
$$

Consider $\Omega(t ; T, \bar{\Omega})$ with $\bar{\Omega} \in B_{j}(T, \bar{\delta})$, and let $\bar{u}(r)$ the corresponding solution of (1.2). According to the invariance property stated in Lemma 3.13, $B_{j}(T, \bar{\delta}) \cap \bar{\Gamma}^{s}(t)=\emptyset$ for every $t \in \mathbb{R}$, so $\bar{u}(r)$ cannot be a fast decay solution; $B_{j}(T, \bar{\delta}) \cap \bar{\Gamma}^{u}(t)=\emptyset$ for every $t \in \mathbb{R}$, so $\bar{u}(r)$ cannot be a regular solution. Moreover, from (3.11) combined with Lemmas 3.1 and 3.3, we infer that $\theta(t ; T, \bar{\Omega}) \in\left(-\frac{\pi}{2}, 0\right)$ for any $t<-\mathcal{T}_{j}$ and $\theta(t ; T, \bar{\Omega}) \in\left(-j \pi-\frac{\pi}{2}, j \pi\right)$ for any $t>\mathcal{T}_{j}$. Since $\bar{u}(r)$ cannot rotate indefinitely as $r \rightarrow \pm \infty$, from Lemma 2.9 we conclude that $\bar{u}(r)$ is a singular-slow decay solution.

More precisely, $\lim _{t \rightarrow-\infty} \theta(t ; T, \bar{\Omega})=\phi^{+}(-\infty), \lim _{t \rightarrow \infty} \theta(t ; T, \bar{\Omega})=\phi^{+}(+\infty)-$ $j \pi$ if $j$ is even, $\lim _{t \rightarrow \infty} \theta(t ; T, \bar{\Omega})=\phi^{-}(+\infty)-(j-1) \pi$ if $j$ is odd.

Arguing exactly as in the proof of Proposition 3.19, we obtain that $\bar{u}(r)$ has exactly $j$ zeroes. This completes the proof.

We now concentrate on regular-slow decay solutions. To this aim, we set

$$
\alpha_{j}(T, \delta):=\left\{\Gamma^{u,+}(U, T) \mid U_{j}(\delta)<U<U_{j}^{*}(T)\right\} .
$$

Proposition 3.23. If $\bar{\Omega} \in \alpha_{j}(T, \bar{\delta})$ then for every $d \in\left(d_{j}, d_{j}^{*}\right)$ the solution $\bar{u}(r, d)$ of (1.2) corresponding to $\Omega(t ; T, \bar{\Omega})$ is regular-slow decay and has exactly $j$ zeroes.

Proof. Let $\bar{u}(r, d)$ be the solution of (1.2) corresponding to $\Omega(t ; T, \bar{\Omega})$. By Lemma 3.13, $\bar{u}(r, d)$ is regular, and $\lim _{t \rightarrow-\infty} \theta(t ; T, \bar{\Omega})=0$.

By definition, $\alpha_{j}(T, \bar{\delta}) \cap \bar{\Gamma}^{s}(T)=\emptyset$, so $\bar{u}(r)$ cannot be a fast decay solution. Observe that the inequalities (3.11) are satisfied by $\bar{\Omega} \in \alpha_{j}(T, \bar{\delta})$, since $\alpha_{j}(T, \bar{\delta}) \in \partial B_{j}(T, \bar{\delta})$. Hence, with the same argument adopted in the proof of Proposition 3.22, we conclude that $\bar{u}(r)$ has slow decay, and $\lim _{t \rightarrow \infty} \theta(t ; T, \bar{\Omega}) \in(\bar{\theta}-j \pi,-j \pi)$.

The thesis easily follows.

Remark 3.24. It might be shown that the connected component of $\boldsymbol{K}_{\boldsymbol{j}}(T)$ containing $B_{j}(T, \bar{\delta})$ is made up by initial conditions corresponding to singular-slow decay solutions with exactly $j$ zeroes, as well as the connected component of $\stackrel{\circ}{\boldsymbol{u}}_{j}^{u}(T)$ containing $\boldsymbol{\alpha}_{j}(T, \bar{\delta})$ is made up by initial conditions corresponding to regular-slow decay solutions with exactly $j$ zeroes, whose endpoints are regular-fast decay solutions.

\section{Proof of Corollary 1.2 and Remark 1.3}

Proof of Corollary 1.2. We begin the proof by explaining the origin of the restrictions on the parameters involved in the Corollary.

\begin{tabular}{|l|l|l|l|l|l|}
\hline 220 & 2546 & $\mathbf{B}$ & $\begin{array}{l}\text { Dispatch: 28/12/2015 } \\
\text { Total pages: 27 } \\
\text { Disk Received } \\
\text { Disk Used } \square\end{array}$ & $\begin{array}{l}\text { Journal: Commun. Math. Phys. } \\
\text { Not Used } \square \\
\text { Corrupted } \square \\
\text { Mismatch } \square\end{array}$ \\
\hline
\end{tabular}


Remark 4.1. The inequality $l<\lambda(q)<s<\eta(q)$ at point 1 is equivalent to

$$
2_{*}<l_{u}:=2 \frac{q+s}{2+s}<2^{*}<2 \frac{q+l}{2+l}=: l_{s} .
$$

Analogously, the inequalities $\lambda\left(q_{1}\right)<s<\eta\left(q_{1}\right)$ and $l<\lambda\left(q_{2}\right)$ at point 2 are equivalent to

$$
2_{*}<l_{u}:=2 \frac{q_{1}+s}{2+s}<2^{*}<2 \frac{q_{2}+l}{2+l}=: l_{s} .
$$

Moreover, the inequalities in (1.11) correspond to

$$
2_{*}<l_{u}:=2 \frac{q_{2}-q_{1}+s_{2}-s_{1}}{2+s_{2}-s_{1}}<2^{*}<2 \frac{q_{2}+l_{2}}{2+l_{2}}=: l_{s} .
$$

Finally, it is easy to show that the inequalities (1.12)-(1.13) are equivalent to

$$
2_{*}<l_{u}:=\max \left\{2 \frac{q_{1}+s_{1}}{2+s_{1}} ; 2 \frac{q_{2}+s_{2}}{2+s_{2}}\right\}<2^{*}<\min \left\{2 \frac{q_{1}+l_{1}}{2+l_{1}} ; 2 \frac{q_{2}+l_{2}}{2+l_{2}}\right\}=: l_{s} \text {. }
$$

Now we are ready to prove the Corollary.

1. When $f$ is of type (1.3) and $k$ satisfies (1.4) under the condition (4.1), it is easy to verify that

$$
g_{l_{u}}(x, t):=k\left(e^{t}\right) e^{-s t} x|x|^{q-2}, \quad g_{l_{s}}(x, t):=k\left(e^{t}\right) e^{-l t} x|x|^{q-2},
$$

implying that $g_{l_{u}}^{-\infty}(x)=A x|x|^{q-2}$ and $g_{l_{s}}^{+\infty}(x)=B x|x|^{q-2}$. Thus, the thesis immediately follows.

2. When $f$ is of type (1.5) and $k$ satisfies (1.4) under the condition (4.2), we obtain

$$
g_{l_{u}}(x, t):=k\left(e^{t}\right) e^{-s t} x|x|^{q_{1}-2} \quad \text { if }|x| \geq e^{\frac{2+s}{q_{1}+s} t},
$$

$$
g_{l_{s}}(x, t):=k\left(e^{t}\right) e^{-l t} x|x|^{q_{2}-2} \quad \text { if }|x| \leq e^{\frac{2+l}{q_{2}+l} t},
$$

from which we deduce that $g_{l_{u}}^{-\infty}(x)=A x|x|^{q_{1}-2}$ and $g_{l_{s}}^{+\infty}(x)=B x|x|^{q_{2}-2}$. The thesis is so achieved.

3. When $f$ is of type (1.6), $k_{i}$ satisfies (1.9) for every $i \in\{1,2\}$ under the condition (4.3), we get

$$
g_{l_{u}}(x, t):=\frac{k_{2}\left(e^{t}\right) x|x|^{q_{2}-2} e^{\alpha_{l_{u}}\left(l_{u}-q_{2}\right) t}}{1+k_{1}\left(e^{t}\right)|x|^{q_{1}} e^{-\alpha_{l_{u}} q_{1} t}} .
$$

Taking into account (1.9)-(1.10), passing to the limit as $t \rightarrow-\infty$, we can conclude that

$$
g_{l_{u}}^{-\infty}(x)=\frac{A_{2}}{A_{1}} x|x|^{q_{2}-q_{1}-2}, \quad \text { since }
$$

$$
s_{2}+\alpha_{l_{u}}\left(l_{u}-q_{2}\right)=s_{1}-q_{1} \alpha_{l_{u}}=\frac{-\left(s_{2}+2\right) q_{1}+s_{1}\left(q_{2}-2\right)}{q_{2}-q_{1}-2}<0 .
$$

\begin{tabular}{|l|l|l|l|l|l|}
\hline 220 & 2546 & $\mathbf{B}$ & $\begin{array}{l}\text { Dispatch: 28/12/2015 } \\
\text { Total pages: 27 } \\
\text { Disk Received } \square \\
\text { Disk Used } \square\end{array}$ & $\begin{array}{l}\text { Journal: Commun. Math. Phys. } \\
\text { Not Used } \square \\
\text { Corrupted } \square \\
\text { Mismatch } \square\end{array}$ \\
\hline
\end{tabular}


Analogously, we obtain

$$
g_{l_{s}}(x, t):=\frac{k_{2}\left(e^{t}\right) x|x|^{q_{2}-2} e^{-l_{2} t}}{1+k_{1}\left(e^{t}\right)|x|^{q_{1}} e^{-\frac{\left(2+l_{2}\right) q_{1}}{q_{2}-2} t}},
$$

from which, according to (1.9)-(1.10), we infer that

$$
g_{l_{s}}^{+\infty}(x)=B_{2} x|x|^{q_{2}-2} .
$$

4. When $f$ is of type (1.7), $k_{i}$ satisfies (1.9) for every $i \in\{1,2\}$ under the condition (4.4), some further calculations lead to the following conclusions

$$
\begin{aligned}
& g_{l_{u}}^{-\infty}(x)= \begin{cases}A_{1} x|x|^{q_{1}-2} & \text { if } l_{u}=2 \frac{q_{1}+s_{1}}{2+s_{1}} \\
A_{2} x|x|^{q_{2}-2} & \text { if } l_{u}=2 \frac{q_{2}+s_{2}}{2+s_{2}} \\
A_{1} x|x|^{q_{1}-2}+A_{2} x|x|^{q_{2}-2} & \text { if } l_{u}=2 \frac{q_{1}+s_{1}}{2+s_{1}}=2 \frac{q_{2}+s_{2}}{2+s_{2}}\end{cases} \\
& g_{l_{s}}^{+\infty}(x)= \begin{cases}B_{1} x|x|^{q_{1}-2} & \text { if } l_{s}=2 \frac{q_{1}+l_{1}}{2+l_{1}} \\
B_{2} x|x|^{q_{2}-2} & \text { if } l_{s}=2 \frac{q_{2}+l_{2}}{2+l_{2}} \\
B_{1} x|x|^{q_{1}-2}+B_{2} x|x|^{q_{2}-2} & \text { if } l_{s}=2 \frac{q_{1}+l_{1}}{2+l_{1}}=2 \frac{q_{2}+l_{2}}{2+l_{2}} .\end{cases}
\end{aligned}
$$

The goal is so achieved.

The next brief paragraph is devoted to prove Remark 1.3, which extends Theorem 1.1 in [6].

Proof of Remark 1.3. Observe that if $f$ is defined as in (1.14), then it satisfies $\boldsymbol{G}_{\mathbf{0}}$ with $l=2^{*}$ and $g_{2 *}(x, t)=\sum_{i=1}^{j} c_{i} x|x|^{q_{i}-2}$.

Since (2.2) is autonomous, it is invariant for translations in $t$. Thus, if $\boldsymbol{x}(t)$ solves (2.2), then $\boldsymbol{x}^{\tau}(t):=\boldsymbol{x}(t-\tau)$ is a solution too. Correspondingly, if $u(r)$ solves (1.2), then $u^{\tau}(r):=u\left(r e^{-\tau}\right) e^{-\alpha_{2} * \tau}$ solves (1.2) too. As a consequence, in the critical case the solutions of (1.2) have a nice scaling property: setting $U(r):=u(r, 1)$, any regular solution $u(r, d)$ satisfies $u(r, d)=U\left(r d^{2 /(n-2)}\right) d$, where $d=e^{-\alpha_{2} * \tau}$. We finally infer that

$$
\begin{aligned}
\mathcal{T}\left(u^{\tau}\right) & =\int_{\mathbb{R}} g_{2^{*}}\left(x^{\tau}(t)\right) e^{\alpha_{2 * t}} d t=\int_{\mathbb{R}} g_{2^{*}}(x(t-\tau)) e^{\alpha_{2 *} t} d t \\
& =e^{\alpha_{2 * \tau}} \int_{\mathbb{R}} g_{2^{*}}(x(t)) e^{\alpha_{2 *} t} d t=d^{-1} \mathcal{T}(u),
\end{aligned}
$$

which completes the proof of the first part of Remark 1.3.

Now, let $G(x)=\sum_{i=1}^{j} \frac{c_{i}}{q_{i}}|x|^{q_{i}}$, then

$$
H(x, y)=\alpha_{2} * x y+\frac{y^{2}}{2}+G(x)
$$

is a first integral for (2.2) and we can draw all the trajectories. Regular solutions of (1.2) correspond to the family of homoclinic trajectories having graph contained in the 0 level set of $H$, see Fig. 1. The second part of Remark 1.3 easily follows.

\begin{tabular}{|l|l|l|l|l|l|}
\hline 220 & 254.6 & $\mathbf{B}$ & $\begin{array}{l}\text { Dispatch: 28/12/2015 } \\
\text { Total pages: 27 } \\
\text { Disk Received } \square \\
\text { Disk Used } \square\end{array}$ & $\begin{array}{l}\text { Journal: Commun. Math. Phys. } \\
\text { Not Used } \square \\
\text { Corrupted } \square \\
\text { Mismatch } \square\end{array}$ \\
\hline
\end{tabular}


Acknowledgements The first author wishes to express her gratitude to the "Centro de Matemática, Aplicações Fundamentais e Investigação Operacional" of the University of Lisbon for its hospitality.

\section{References}

1. Bidaut-Véron, M.F.: Local and global behavior of solutions of quasilinear equations of Emden-Fowler type. Arch. Rational Mech. Anal. 107, 293-324 (1989)

2. Bamon, R., Flores, I., Del Pino, M.: Ground states of semilinear elliptic equations: a geometric approach. Ann. Inst. Henry Poincaré 17, 551-581 (2000)

3. Battelli, F., Johnson, R.: Singular ground states of the scalar curvature equation in $\mathbb{R}^{n}$. Diff. Int. Equ. 14, 123-139 (2000)

4. Capietto, A., Dambrosio, W., Zanolin, F.: Infinitely many radial solutions to a boundary value problem in a ball. Ann. Mat. Pura Appl. 179, 159-188 (2001)

5. Cheng, K.S., Chern, J.L.: Existence of positive entire solutions of some semilinear elliptic equations. J. Diff. Equ. 98, 169-180 (1992)

6. Chern, J.L., Chen, Z.Y., Tang, Y.L.: Uniqueness of finite total curvatures and the structure of radial solutions for nonlinear elliptic equations. Trans. Am. Math. Soc. 363(6), 3211-3231 (2011)

7. Chern, J.L., Yanagida, E.: Structure of the sets of regular and singular radial solutions for a semilinear elliptic equation. J. Differ. Equ. 224, 440-463 (2006)

8. Coffman, C.V., Ullrich, D.F.: On the continuation of solutions of a certain non-linear differential equation. Monatsh. Math. 71, 385-392 (1967)

9. Damascelli, L., Pacella, F., Ramaswamy, M.: Symmetry of ground states of p-Laplace equations via the Moving Plane Method. Arch. Rat. Mech. Anal. 148, 291-308 (1999)

10. Erbe, L., Tang, M.: Structure of positive radial solutions of semilinear elliptic equations. J. Differ. Equ. 133, 179-202 (1997)

11. Felmer, P., Quaas, A., Tang, M.: On the complex structure of positive solutions to Matukuma-type equations. Ann. Inst. H. Poincaré Anal. Non Linéaire 26, 869-887 (2009)

12. Fowler, R.H.: Further studies of Emden's and similar differential equations. Q. J. Math. 2, 259-288 (1931)

13. Franca, M.: Classification of positive solution of $p$-Laplace equation with a growth term. Arch. Math. (Brno) 40(4), 415-434 (2004)

14. Franca, M.: Fowler transformation and radial solutions for quasilinear elliptic equations. Part 1: the subcritical and supercritical case. Can. Math. Appl. Quart. 16, 123-159 (2008)

15. Franca, M.: Structure theorems for positive radial solutions of the generalized scalar curvature equation. Funkcialaj Ekvacioj 52, 343-369 (2009)

16. Franca, M.: Fowler transformation and radial solutions for quasilinear elliptic equations. Part 2: nonlinearities of mixed type. Ann. Mat. Pura Appl. 189, 67-94 (2010)

17. Franca, M.: Positive solutions for semilinear elliptic equations: two simple models with several bifurcations. J. Dyn. Differ. Equ. 23, 573-611 (2011)

18. Franca, M.: Positive solutions of semilinear elliptic equations: a dynamical approach. Differ. Int. Equ. 26, 505-554 (2013)

19. Franca, M., Johnson, R.: Ground states and singular ground states for quasilinear partial differential equations with critical exponent in the perturbative case. Adv. Nonlinear Stud. 4, 93-120 (2004)

20. García-Huidobro, M., Manasevich, R., Yarur, C.: On the structure of positive radial solutions to an equation containing $p$-Laplacian with weights. J. Differ. Equ. 223, 51-95 (2006)

21. Gidas, B., Ni, W.M., Nirenberg, L.: Symmetry of positive solutions of nonlinear elliptic equations in $\mathbb{R}^{n}$. Adv. Math. Suppl. Stud. 7, 369-403 (1981)

22. Hale, J.: Ordinary Differential Equation. Pure Appl. Math. 21, (1980)

23. Hirsch, M., Pugh, C., Shub, M.: Invariant Manifolds, Lecture Notes in Math., vol. 583. Springer-Verlag, New York (1977)

24. Johnson, R.: Concerning a theorem of Sell. J. Differ. Equ. 30, 324-339 (1978)

25. Johnson, R., Pan, X.B., Yi, Y.F.: Singular ground states of semilinear elliptic equations via invariant manifold theory. Nonlinear Anal. Th. Meth. Appl. 20, 1279-1302 (1993)

26. Johnson, R., Pan, X.B., Yi, Y.F.: The Melnikov method and elliptic equation with critical exponent. Indiana Math. J. 43, 1045-1077 (1994)

27. Jones, C., Küpper, T.: On the infinitely many solutions of a semilinear elliptic equation. SIAM J. Math. Anal. 17(4), 803-835 (1986)

28. Kabeya, Y., Yanagida, E., Yotsutani, S.: Existence of nodal fast-decay solutions to $\operatorname{div}\left(|\nabla u|^{m-2} \nabla u\right)+$ $K(|x|)|u|^{q-1} u=0$ in $\mathbb{R}^{n}$. Differ. Integral Equ. 9, 981-1004 (1996)

29. Kawano, N., Yanagida, E., Yotsutani, S.: Structure theorems for positive radial solutions to $\Delta u+$ $K(|x|) u^{q}=0$ in $\mathbb{R}^{n}$. Funkcialaj Ekvacioj 36, 557-579 (1993)

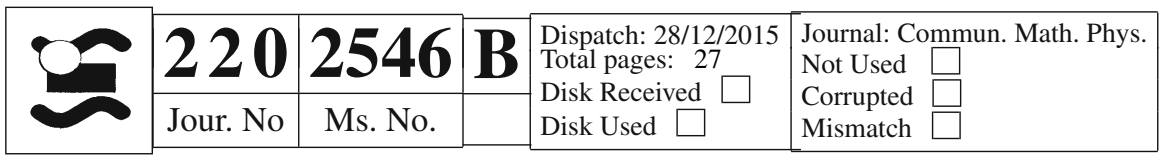


30. Morishita, H., Yanagida, E., Yotsutani, S.: Structure of positive radial solutions including singular solutions to Matukuma's equation. Commun. Pure Appl. Anal. 4(4), 871-888 (2005)

31. Naito, Y.: Bounded solutions with prescribed numbers of zeros for the Emden-Fowler differential equation. Hiroshima Math. J. 24, 177-220 (1994)

32. Ni, W.M.: On the elliptic equation $\Delta u+K(x) u^{\frac{n+2}{n-2}}=0$, its generalization, and applications in geometry. Indiana Univ. Math. J. 31, 493-529 (1982)

33. Ni, W.M., Serrin, J.: Nonexistence theorems for quasilinear partial differential equations. Rend. Circolo Mat. Palermo (Centenary Supplement), Ser. II 8, 171-185 (1985)

34. Ni, W.M., Yotsutani, S.: On Matukuma's equation and related topics. Proc. Jpn. Acad. Ser. A 62, 260-263 (1986)

35. Ni, W.M., Yotsutani, S.: Semilinear elliptic equations of Matukuma-type and related topics. Jpn. J. Appl. Math. 5, 1-32 (1988)

36. Papini, D.: Boundary value problems for second order differential equations with superlinear terms: a topological approach. Ph.D. Thesis, S.I.S.S.A, Trieste (2000)

37. Pucci, P., García-Huidobro, M., Manasevich, R., Serrin, J.: Qualitative properties of ground states for singular elliptic equations with weights. Ann. Mat. Pura Appl., 185, S205-S243 (2006)

38. Serrin, J., Zou, H.: Symmetry of ground states of quasilinear elliptic equations. Arch. Rational Mech. Anal. 148, 265-290 (1985)

39. Yanagida, E.: Structure of radial solutions to $\Delta u+K(|x|)|u|^{p-1} u=0$ in $\mathbb{R}^{n}$. SIAM J. Math. Anal. 27(3), 997-1014 (1996)

40. Yanagida, E., Yotsutani, S.: Classification of the structure of positive radial solutions to $\Delta u+K(|x|) u^{p}=0$ in $\mathbb{R}^{n}$. Arch. Rational Mech. Anal. 124, 239-259 (1993)

41. Yanagida, E., Yotsutani, S.: Existence of nodal fast-decay solutions to $\Delta u+K(|x|)|u|^{p-1} u=0$ in $\mathbb{R}^{n}$. Nonlinear Anal. 22, 1005-1015 (1994)

42. Yanagida, E., Yotsutani, S.: Existence of positive radial solutions to $\Delta u+K(|x|) u^{p}=0$ in $\mathbb{R}^{n}$. J. Differ. Equ. 115, 477-502 (1995)

Communicated by W. Schlag 